\title{
BLOCK FOREST (ROSO PENDULINAE-TILIETUM CORDATAE), A NEW FOREST COMMUNITY OF THE CARPATHIAN BASIN (CEROVÁ VRCHOVINA, SLOVAKIA)
}

\author{
J. CSIKY ${ }^{1}$, B. KEVEY² and A. BORHIDI ${ }^{3}$ \\ ${ }^{1}$ Research Group for Biological Adaptation of the Hungarian Academy of Sciences \\ University of Pécs, H-7601 Pécs, Ifjúság útja 6, Hungary; E-mail: moon@ttk.pte.hu \\ ${ }^{2}$ Dept Botany, University of Pécs, H-7601 Pécs, Ifjúság útja 6, Hungary \\ ${ }^{3}$ Inst. Ecology and Botany, Hungarian Academy of Sciences, H-2163 Vácrátót, Hungary
}

(Received 10 August, 2000)

\begin{abstract}
A new plant association (Roso pendulinae-Tilietum cordatae), similar to the Poo nemoralisTilietum cordatae Firbas et Sigmond 1928 (Central European siliceous block field forest), has been described. This forest community develops on the periglacial block fields of southern Slovakia (Cerová Vrchovina, syn.: Nógrád-Gömör basalt region). The community has been separated from the Mercuriali-Tilietum Zólyomi et Jakucs 1958 common in the submontane belt of the Pannonicum using phytocoenological comparative analyses. The ecological demand and the species combination clearly distinguish this association from the other forest communities described earlier from northern periglacial block fields of Hungary (e.g. Mercuriali-Tilietum, Tilio tomentosae-Fraxinetum orni). The absence of the early vernal aspect (e.g. Adoxa moschatellina, Corydalis cava, C. solida, Galanthus nivalis, Isopyrum thalictroides, etc.), of submediterranean shrubs, such as Cornus mas, Staphylea pinnata, Viburnum lantana, as well as of Fraxinus excelsior and F. ornus is remarkable at all sites. On the other hand, the block field forest contains species characteristic of continental forest-steppes (e.g. Cotoneaster matrensis, Euonymus verrucosus, Spiraea media, etc.), coexisting with montane elements (e.g. Rosa pendulina, Sambucus racemosa, Sorbus aucuparia, Dryopteris dilatata, etc.). These habitats are suitable for the abundant growth of mosses, hepatics and lichens, especially Andreaea rupestris, Cladonia rangiferina, C. squamosa, Dicranum tauricum, Diploschistes scruposus, Hedwigia ciliata, Polytrichum piliferum, Tritomaria quinquedentata, etc. The herb layer is dominated by ferns, such as Asplenium septentrionale, A. trichomanes, A. $\times$ alternifolium, Cystopteris fragilis, Dryopteris carthusiana, D. dilatata, D. filix-mas and Polypodium vulgare.
\end{abstract}

Key words: basalt, block forest, Cerová Vrchovina, Mercuriali-Tilietum, Northern Hungarian Mts, periglacial block fields, phytosociological system 


\section{INTRODUCTION}

\section{Phytogeographical situation}

The Nógrád-Gömör basalt region (approx. $430 \mathrm{~km}^{2}$ ) is situated along the central part of the Hungarian-Slovakian border. According to modern phytogeographical divisions, the territory belongs to the sector of the Northern Hungarian Mts (Matricum) within the Pannonian flora province (Pannonicum). Slovak botanists define the area as part of the IpelRimavska brazda (Dostál and Cervenka 1991), while the Hungarian region is considered as part of the Agriense (Soó 1960).

The area is to be considered as one of the northernmost representative of the Pannonian flora, being also in close contact with the Carpathian province (Michalko 1987). It is supposed that during the Late Holocene (subboreal period) the vegetation of the area was dominantly mesophilous beech wood of a more accentuated Carpathian character. This assumption is supported by the occurrence of some relict species, such as Dentaria glandulosa, Matteuccia struthiopteris, Prenanthes purpurea, Petasites albus, etc., living in the submontane belt of the basalt area.

From geomorphological aspect the basalt is characterized by high relief, where the special flora of the habitats of various morphological features (e.g. periglacial block fields) refers to the different stages of the vegetation history (e.g. Andreaea rupestris, Spiraea media, Rosa spinosissima, $R$. pendulina, Dryopteris dilatata, Sambucus racemosa and Valeriana stolonifera).

\section{Ecological survey}

In spite of the geological diversity (rhyolite, andesite, basalt, sandstone, etc.) the basalt outcrops (basalt plateaux, basalt volcanic cones) and the underlying Oligocene-Miocene sediments define the land morphology of the region. This structure resulted in varied topography (Jugovics 1971, Horváth et al. 1997). The extent of the periglacial block fields in the Nógrád-Gömör basalt region is unusual in the submontane belt of the region. The basalt has undergone rapid physical weathering due to its special characteristics (olivin content, horizontal and vertical jointing) and the intensive frost shattering during the Pleistocene (Jugovics 1971, Gábris 1995).

At the higher altitudes climate shows a montane character (mean temperature in January: from -2.5 to $-6^{\circ} \mathrm{C}$, the same in July: from 17 to $19.5^{\circ} \mathrm{C}$, annual precipitation: $600-850 \mathrm{~mm}$ ) (Michalko 1987). The majority of the 
block fields is located in the altitude of $400-600 \mathrm{~m}$ asl. belonging to the oak-hornbeam (Carici pilosae-Carpinetum) and the submontane beech (Melitti-Fagetum) zone here.

The large (basalt) blocks create a special microclimate on the scree slopes (Kun 1998). The emerging rock surfaces act as edaphic deserts (arid and warm, arid and cold), while the cavities between them act as frost traps (humid and cold). Under the debris cover the snow remains until May at the entrance of the secondary caves. Extensive block fields and blocks of considerable size are found only along the rims of the basalt plateaux and larger basalt cones. At some places contact springs issue under the block fields. Due to these springs and the secondary caves a special cold and humid microclimate prevails between the blocks, which has preserved relict animals, like Salamandra salamandra. This amphibian is abundant only on these slopes and along some streams in the Nógrád-Gömör basalt region.

These edaphic conditions cannot be found on narrow ridges as it was written in earlier papers (Kun 1998).

At places, where the regolith is too thick (more than $1 \mathrm{~m}$ in diameter) for the roots of higher plants, there are extensive gaps. This competition-free habitat is suitable for lichens, mosses and some ferns. Some very rare species live in these gaps such as the glacial relict moss of the Hungarian submontane region, Andreaea rupestris, the montane fern species, Dryopteris dilatata and some sporadic hybrid taxa, such as Asplenium $\times$ alternifolium and Rosa $\times$ reversa. The largest Hypno-Polypodietum stands are growing here, forming a mosaic pattern under the canopy as well.

The microclimatic bipolarity of these habitats and the special transitional situation of the Nógrád-Gömör basalt region between the phytogeographical provinces Pannonicum and Carpaticum preserved relict species from different stages of the vegetation history (Boreal and subboreal periods).

Usually the block forest stands are in contact with submontane beech woods, Carpathian oak-hornbeam woods and in some cases with stands of the Tilio-Fraxinetum. On basalt cones block forests occur only on the northern slopes, while on basalt plateaux in all exposition.

Some periglacial block fields are also present at lower altitudes adjoining to xero-mesophilous oak woods, but in their flora the characteristic Tilio-Acerion and Fagetalia elements are represented by a lower number of species and decreasing abundance. It is assumed that the fall in the selectivity and relict preserving capacity of the block fields is in accordance with 
the lower altitude, higher temperature, the smaller block size and the decreasing thickness of the debris cover.

\section{Phytosociological antecedents}

Several previous phytosociological descriptions have been published about scree slopes and ridges in the Carpathian Basin. The lime scree forests on northern steep rocky slopes have been differentiated by Zólyomi (1958) as Mercuriali-Tilietum. The typical stand comes from the dachstein limestone scree slope of the Buda Hills. The Mercuriali-Tilietum definitely differs from Tilio-Fraxinetum excelsioris Zólyomi (1934) 1967 described earlier from crests, ridges and rocky peaks in the absence of continental elements (e.g. Spiraea media, Carex brevicollis) and the increasing ratio of Fagetalia (e.g. Acer pseudoplatanus) and submediterranean species (e.g. Scutellaria columnae). Based on these differences, they were classified into separate phytosociological classes (Table 1).

As for physiognomy, lime-dominated scree forests are similar along the Transdanubian and Northern Hungarian Mts: the canopy layer is built up by tree species well adapted to the rocky substrate such as lime, maple and ash with their curved trunks forming bunches, by the shrub layer of

Table 1

The treated communities in the actual syntaxonomic system according to Borhidi (1996) and Mucina et al. (1993)

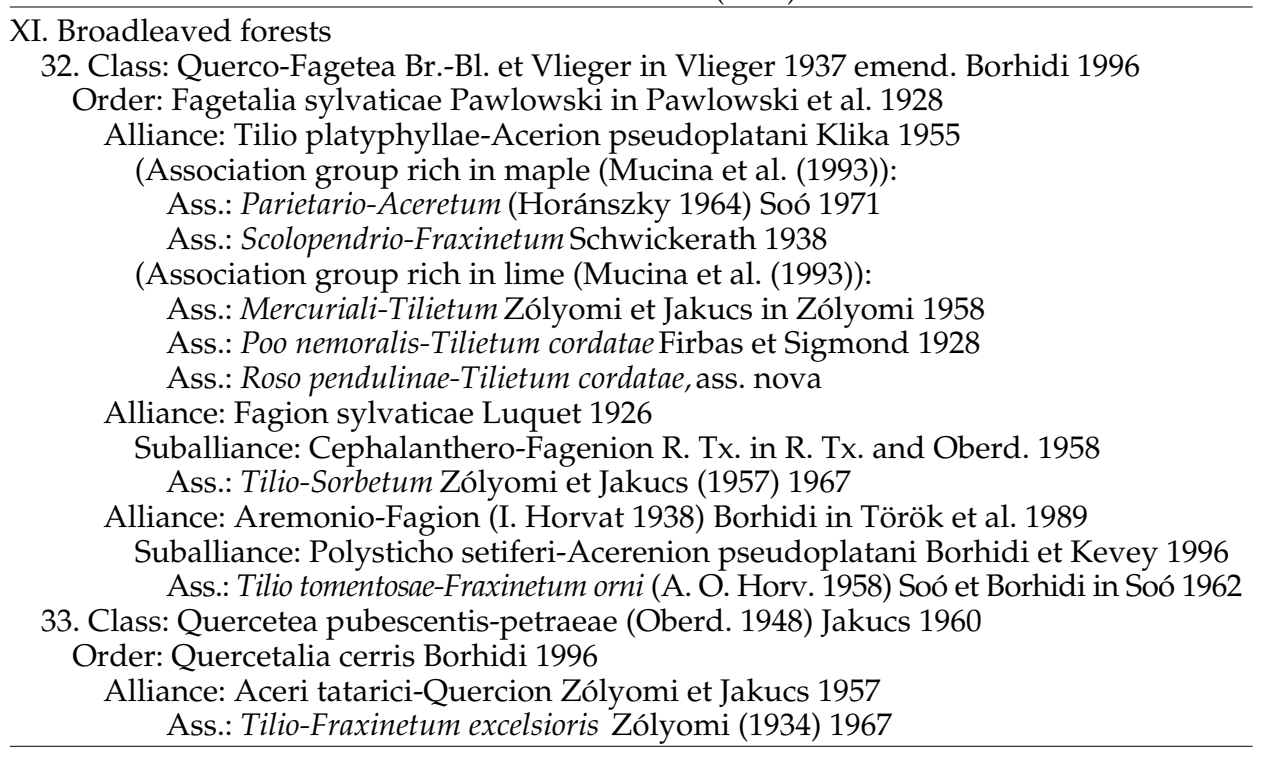


Cornus mas and Staphylea pinnata, while the herb layer is characterized by a striking vernal aspect. Due to atlantic and mediterranean influences the Tilio tomentosae-Fraxinetum orni (A. O. Horv. 1958) Soó et Borhidi in Soó 1962 of the Mecsek and Villány Mts is rich in submediterranean and subatlantic elements, such as Tilia tomentosa in the canopy, Ruscus aculeatus in the shrub layer and Aremonia agrimonoides, Asperula taurina, Doronicum orientale, Ruscus hypoglossum, Helleborus odorus, Chaerophyllum aureum in the herb layer.

In spite of considerable overlap in the flora, the physiognomy of the gorge forests (e.g. Scolopendrio-Fraxinetum Schwickerath 1938, Parietario-Aceretum (Horánszky 1964) Soó 1971) and lime-dominated scree forests differs well in the height and habit of trees. Mucina et al. (1993) divided the Tilio platyphyllae-Acerion pseudoplatani Klika 1955 into two groups (Table 1): one dominated by maple (e.g. gorgeforests) and another one rich in lime (e.g. block forests).

Roso pendulinae-Tilietum has many common species with the Tilio-Sorbetum Zólyomi et Jakucs (1957) 1967, described from the limestone escarpments of the Bükk Mts. In contrast with scree forests, the stands of TilioSorbetum grow on steep rocks, their canopy is lower, the shrub layer is mostly sparse and several calciphilous differential species characterize the herb and moss layers.

Mercuriali-Tilietum stands are widespread in the Hungarian mountains, as reported in numerous papers (Jakucs 1961, Fekete and Járai-Komlódi 1962, Fekete 1964, Horánszky 1964, Szujkó-Lacza 1967, Zólyomi 1967, Isépy 1968, Kovács 1968, Simon 1977, Debreczy 1981, Bartha et al. 1995, Kovács and Takács 1995, Vojtkó 1990,1995, 1996, Fekete et al. 1997). Due to the frequent occurrence of transitional stands, many sampled plots were treated as rocky beech forest, gorge forest or typical Mercuriali-Tilietum (Fekete 1964, Kovács 1968, Simon 1977, Szmorad 1994, Nagy 1999).

Lime block forests (Aceri-Tilietum, Tilia cordata-Poa nemoralis, Acero-Tilietum, Acereto-Tilietum, etc.) growing on siliceous substrate have often been mentioned in German studies (Firbas and Sigmond 1928, Bartsch and Bartsch 1940, Oberdorfer 1957, Mayer 1984). These forests are typical edaphic, intrazonal associations in the submontane beech and oak-hornbeam belts, with a characteristic physiognomy, structure and composition influenced mostly by the morphology of the surface (periglacial "Felsenmeer" with large blocks).

Due to their successful generative and vegetative reproduction and distribution strategy (spreading by wind and animals) the characteristic 
tree and shrub species tolerate the talus creep and are well adapted to the shallow soil. In case of some species (e.g. Tilia cordata) self-saprophytism might have been advantageous for the roots to grow through unfavourable soil layers (Sen 1961).

The phytosociological consideration of these lime block forest associations has not always been uniform. According to their diffuse, sporadic occurrence in Central Europe, their flora is influenced by the elements of the neighbouring phytogeographical provinces. In the Swiss Jura and around Lake Boden they are rich in submediterranean and subatlantic elements (e.g. Tamus communis, Helleborus foetidus, Ilex aquifolium, Euonymus latifolius, Asperula taurina, etc.), while in the Hungarian mountains more continental elements are present (e.g. Spiraea media, Euonymus verrucosus, etc.). The floristical differences point to the diverse historical development of a given stand.

Mucina et al. (1993) separated a part of the relevés from the block forests considering them as Poo nemoralis-Tilietum cordatae Firbas et Sigmond 1928 ("Silikat-Blokkhalden-Lindenwald"). Since block forests have not yet been reported from the Hungarian mountains, Hungarian botanists treated these stands as gorge forests or montane beech forests. The presence of some species characteristic of gorge forest, such as Lunaria rediviva, Polystichum spp. supports this decision. Because of the high altitude and humidity of these sites, their soil type is similar to that of the gorge forests. Where the debris of the block forest is so thick that permits only a loose closing of the canopy, the characteristic species of the gorge forest disappear and they will replaced by other elements unusual in them.

The edaphic associations mentioned above are (relatively) easily distinguishable with the help of the following table (Table 2).

\section{MATERIAL AND METHODS}

Sampling plots were studied with the square method of the ZürichMontpellier school according to Braun-Blanquet (1928). All the sampling plots were studied twice a year (because of the absence of the vernal aspect only the summer aspect was recorded). Table ordination of the species (Raunkiaer's life form categories, flora elements, Borhidi's ecological indicator values, phytosociological characterization) and relevés calculations based on the presence and absence of the species were carried out with the software developed by Kevey and Hirmann (Kevey 1993, 1997, Kevey and 
Table 2

Differences between the forest associations of the rocky habitats in the Pannonicum

\begin{tabular}{|c|c|c|c|c|}
\hline & $\begin{array}{l}\text { Rocky steppe } \\
\text { forest }\end{array}$ & $\begin{array}{l}\text { Lime-dominated } \\
\text { scree forest }\end{array}$ & Block forest & Gorge forest \\
\hline $\begin{array}{l}\text { Occurrence } \\
\text { (veg. belt) }\end{array}$ & oak and beech & beech & oak and beech & beech \\
\hline Geomorphology & $\begin{array}{l}\text { rocky peak, } \\
\text { ridge }\end{array}$ & scree slope & $\begin{array}{l}\text { "Felsenmeer", } \\
\text { block field }\end{array}$ & $\begin{array}{l}\text { valley, steep } \\
\text { slope }\end{array}$ \\
\hline $\begin{array}{l}\text { Substrate } \\
\text { (bed-rock) }\end{array}$ & $\begin{array}{l}\text { limestone, } \\
\text { volcanic }\end{array}$ & $\begin{array}{l}\text { limestone, } \\
\text { volcanic }\end{array}$ & volcanic & $\begin{array}{l}\text { limestone, } \\
\text { andesite }\end{array}$ \\
\hline Soil type & $\begin{array}{l}\text { rendzina, } \\
\text { "mullranker" }\end{array}$ & black humus & ("mullranker") & black humus \\
\hline Canopy, cover & open & close & open & close \\
\hline Canopy, height & $\begin{array}{l}\text { low } \\
(10-15 \mathrm{~m})\end{array}$ & $\begin{array}{l}\text { middle high } \\
(15-25 \mathrm{~m})\end{array}$ & $\begin{array}{l}\text { middle high } \\
(10-20 \mathrm{~m})\end{array}$ & $\begin{array}{l}\text { high (20-35 } \\
\text { m) }\end{array}$ \\
\hline Physiognomy & $\begin{array}{l}\text { curved trunk } \\
\text { forming bunch }\end{array}$ & $\begin{array}{l}\text { curved trunk } \\
\text { forming bunch }\end{array}$ & $\begin{array}{l}\text { curved trunk } \\
\text { forming bunch }\end{array}$ & timber trees \\
\hline Shrub layer & well developed & well developed & well developed & undeveloped \\
\hline Herb layer & $\begin{array}{l}\text { striking vernal } \\
\text { aspect }\end{array}$ & $\begin{array}{l}\text { striking vernal } \\
\text { aspect }\end{array}$ & $\begin{array}{l}\text { absence of vernal } \\
\text { aspect }\end{array}$ & $\begin{array}{l}\text { striking ver- } \\
\text { nal aspect }\end{array}$ \\
\hline Moss layer & subordinated & well developed & $\begin{array}{l}\text { very well devel- } \\
\text { oped }\end{array}$ & $\begin{array}{l}\text { well } \\
\text { developed }\end{array}$ \\
\hline $\begin{array}{l}\text { Selective } \\
\text { factors }\end{array}$ & exposed rocks & $\begin{array}{l}\text { mobile rocks } \\
\text { (scree) }\end{array}$ & $\begin{array}{l}\text { blocks (heat and } \\
\text { frost) }\end{array}$ & $\begin{array}{l}\text { wet rocky } \\
\text { slopes, frost }\end{array}$ \\
\hline
\end{tabular}

Borhidi 1998). According to the synthetic tables of the forests (Zólyomi 1958, Isépy 1968, Kovács 1968, Simon 1977, Kevey ined., Nagy ined.) the characteristic species combination of the Roso pendulinae-Tilietum cordatae was stated based on the constancy of the differential species.

Based on relevés made by Kevey (ined.) (Keszthely Mts, Bakony Mts, Gerecse Mts), Kovács (1968) and Simon (1977), the associations of the Hungarian scree slopes were compared with the block forests of the Cerová Vrchovina using cluster analysis (Statistica for Windows). Within the phytosociological table species are grouped according to their position in the syntaxonomic system following the catalogue of Soó elaborated for the Hungarian Flora, amplified and critically revised by Borhidi $(1993,1995)$ (see also Horváth et al. 1995). For the vascular plant names Simon (1992), for the moss and hepatic names Corley et al. (1981), Corley and Crundwell (1991), Grolle (1983), for the lichen names Verseghy (1994), for the syntaxonomic 
nomenclature Borhidi (1996), Mucina et al. (1993) and Pott (1995) were considered.

\section{RESULTS}

Distinction and characterization of the

Roso pendulinae-Tilietum cordatae ass. nova

Mercuriali-Tilietum and Roso pendulinae-Tilietum cordatae relevés originating from four Hungarian mountains (Keszthely Mts, Bakony Mts, Mátra Mts and Cerová Vrchovina) were used to characterize the block forests of the Nógrád-Gömör basalt region. In the block forests xerophilous grassland elements (Festuco-Bromea), acidophilous oak wood species (Quercetea robori-petraeae) and xerophilous oak forest plants (Quercetea pubescentis-petraeae) are represented with higher frequency (Fig. 1). The role of these elements keeps increasing starting from the Transdanubian Mts up to the Northern Hungarian Mts. The Herbosa/Lignosa ratio is also the highest in the block forest stands. It may suggest strong geological influence, as

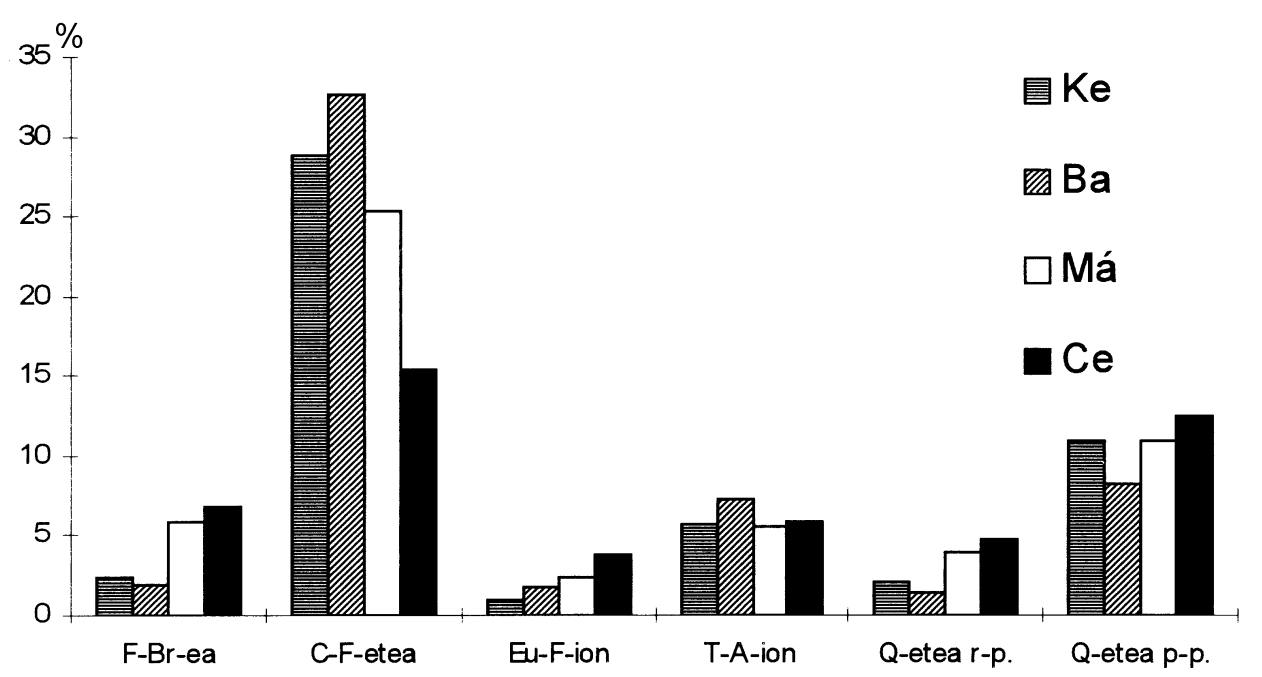

Fig. 1. Frequency of the phytosociological groups. F-Br-ea: Festuco-Bromea, C-F-etea: Carpino-Fagetea, Eu-F-ion: Eu-Fagion, T-A-ion: Tilio-Acerion, Q-etea r-p.: Quercetea roboripetraeae, Q-etea p-p.: Quercetea pubescentis-petraeae, Ke: Keszthely Mts, Ba: Bakony Mts, Má: Mátra Mts, Ce: Cerová Vrchovina 
periglacial weathering was more intensive on the volcanic rocks of the Northern Hungarian Mts, producing coarser-grained debris than on the carbonates of the Transdanubian Mts (Kun 1998). Due to the thick debris closed canopy could not form, consequently, light plants and xerophilous species had opportunity to colonize abundantly here (e.g. Asplenium septentrionale, Sedum maximum and Sedum acre). In accordance with this, mesophilous forest (Carpino-Fagetea) elements are scarce in the block forests of the Nógrád-Gömör basalt region, since they are unable to survive in directly lighted and dry habitats. The microclimatic conditions of the cavities between the blocks and the shade under the patches of canopy might have favoured the survival of these subboreal phase elements in the collin-submontane region of the Pannonicum.

Comparing twelve synthetic tables from the following mountains Keszthely Mts (Kevey ined.), Bakony Mts (Kevey ined.), Vértes Mts (Isépy 1968), Gerecse Mts (Kevey ined.), Buda Hills (Zólyomi 1958), Börzsöny Mts (Nagy ined.), Mátra Mts (Kovács 1968), Zemplén Mts (Simon 1977), Cerová Vrchovina (Csiky ined.), Swiss Jura (Oberdorfer 1957), Frankenwald (Oberdorfer 1957), Schwarzwald (Oberdorfer 1957), conspicuous similarities were found among the frequent species of the scree and block forests (Table 3). The dominant species in the canopy are Tilia platyphyllos and Tilia cordata. Euonymus verrucosus plays a considerable role in the shrub layer in the Pannonicum, while it is absent in the relevés recorded west from the Carpathians. Poa nemoralis and Dryopteris filix-mas are usually present in the herb layer with a high constancy.

There are important and characteristic differencies between the Roso pendulinae-Tilietum cordatae and the Mercuriali-Tilietum. Some species lacking from the block forests of the Cerová Vrchovina are present in the lime-dominated scree forests with high constancy, such as Fraxinus excelsior in the canopy and Staphylea pinnata and Cornus mas in the shrub layer. Viburnum lantana is absent from (the scree forests of) the Northern Hungarian Mts. The species of the vernal aspect and the characteristic species of the Mercuriali-Tilietum, such as Adoxa moschatellina, Mercurialis perennis, Omphalodes scorpioides, Gagea minima, Geranium lucidum fail completely from the block forests of the Nógrád-Gömör basalt region. Melica uniflora, dominant and widespread in other scree habitats, is sporadic here. The mentioned species (e.g. Melica uniflora, Anemone ranunculoides, Corydalis cava, C. solida, Galanthus nivalis, etc.) play a decreasing role from the Transdanubian Mts to the Northern Hungarian Mts. Most of the characteristic 


\section{Table 3}

The constancy values of common and differential species in Roso pendulinae-Tilietum cordatae (9), Mercuriali-Tilietum (1-8) and "Aceri-Tilietum" (Poo nemoralis-Tilietum

\begin{tabular}{|c|c|c|c|c|c|c|c|c|c|c|c|c|}
\hline Common species & 1 & 2 & 3 & 4 & 5 & 6 & 7 & 8 & 9 & 10 & 11 & 12 \\
\hline Tilia cordata & III & $\mathrm{I}$ & $\mathrm{V}$ & $\mathrm{I}$ & - & III & II & III & III & $\mathrm{I}$ & III & - \\
\hline Tilia platyphyllos & V & V & V & $\mathrm{V}$ & $\mathrm{V}$ & V & III & II & III & $\mathrm{V}$ & II & III \\
\hline Euonymus verrucosus & $\mathrm{V}$ & II & IV & IV & $\mathrm{V}$ & III & III & II & $\mathrm{V}$ & - & - & - \\
\hline Dryopteris filix-mas s. str. & $\mathrm{V}$ & IV & - & IV & - & IV & IV & II & $\mathrm{V}$ & $\mathrm{I}$ & II & II \\
\hline Melica uniflora & III & IV & IV & $\mathrm{V}$ & $\mathrm{V}$ & V & III & III & $\mathrm{I}$ & III & - & I \\
\hline Poa nemoralis & IV & IV & II & $\mathrm{V}$ & - & IV & $\mathrm{V}$ & IV & $\mathrm{V}$ & III & IV & II \\
\hline Polypodium vulgare & II & III & II & III & III & III & - & III & $\mathrm{V}$ & - & - & - \\
\hline Sedum maximum & III & III & II & IV & $\mathrm{V}$ & III & $\mathrm{V}$ & III & $\mathrm{V}$ & - & - & - \\
\hline Negative differential species & 1 & 2 & 3 & 4 & 5 & 6 & 7 & 8 & 9 & 10 & 11 & 12 \\
\hline Fraxinus excelsior & V & V & $\mathrm{V}$ & $\mathrm{V}$ & $\mathrm{V}$ & V & II & II & - & $\mathrm{V}$ & III & III \\
\hline Cornus mas & IV & III & $\mathrm{V}$ & III & $\mathrm{V}$ & II & - & I & - & - & - & - \\
\hline Staphylea pinnata & $\mathrm{V}$ & IV & - & II & $\mathrm{V}$ & I & - & II & - & - & - & - \\
\hline Viburnum lantana & $\mathrm{I}$ & II & $\mathrm{I}$ & - & IV & - & - & - & - & IV & $\mathrm{I}$ & I \\
\hline Aconitum vulparia & - & IV & - & $\mathrm{V}$ & I & - & - & - & - & I & - & - \\
\hline Adoxa moschatellina & III & II & - & $\mathrm{V}$ & III & I & I & - & - & - & - & - \\
\hline Allium ursinum & $\mathrm{V}$ & V & - & $\mathrm{V}$ & - & - & - & - & - & - & - & - \\
\hline Anemone ranunculoides & II & IV & III & $\mathrm{V}$ & $\mathrm{V}$ & III & II & - & - & - & - & - \\
\hline Anthriscus nitida & - & III & - & - & - & - & - & - & - & - & - & - \\
\hline Asarum europaeum & II & III & I & III & III & - & - & III & - & I & - & - \\
\hline Corydalis cava & V & $\mathrm{V}$ & III & $\mathrm{V}$ & $\mathrm{V}$ & II & II & - & - & - & - & - \\
\hline Corydalis intermedia & - & V & III & $\mathrm{V}$ & - & - & - & - & - & - & - & - \\
\hline Corydalis solida & V & - & - & $\mathrm{V}$ & $\mathrm{V}$ & III & II & I & - & - & - & - \\
\hline Dentaria bulbifera & - & IV & I & $\mathrm{V}$ & $\mathrm{I}$ & II & I & I & - & - & - & - \\
\hline Gagea lutea & IV & III & - & $\mathrm{V}$ & II & II & - & - & - & - & - & - \\
\hline Gagea minima & - & - & - & - & IV & I & I & - & - & - & - & - \\
\hline Galanthus nivalis & III & $\mathrm{V}$ & $\mathrm{I}$ & $\mathrm{V}$ & IV & II & II & - & - & - & - & - \\
\hline Geranium lucidum & - & - & - & III & IV & II & - & - & - & - & - & - \\
\hline Geranium phaeum & I & III & - & I & - & - & I & - & - & - & - & - \\
\hline Isopyrum thalictroides & - & IV & - & III & III & I & II & - & - & - & - & - \\
\hline Melica uniflora & III & IV & IV & $\mathrm{V}$ & $\mathrm{V}$ & V & III & III & $\mathrm{I}$ & III & - & - \\
\hline Mercurialis perennis & IV & II & $\mathrm{V}$ & $\mathrm{V}$ & IV & II & IV & III & - & $\mathrm{V}$ & III & III \\
\hline Omphalodes scorpioides & - & II & - & $\mathrm{V}$ & I & - & I & - & - & - & - & - \\
\hline
\end{tabular}




\begin{tabular}{|c|c|c|c|c|c|c|c|c|c|c|c|c|}
\hline Positive differential species & 1 & 2 & 3 & 4 & 5 & 6 & 7 & 8 & 9 & 10 & 11 & 12 \\
\hline Betula pendula & - & - & - & - & - & - & - & $\mathrm{I}$ & IV & - & - & - \\
\hline Populus tremula & - & - & - & - & - & - & - & $\mathrm{I}$ & III & - & - & - \\
\hline Salix caprea & - & - & - & - & - & - & - & - & II & - & - & - \\
\hline Sorbus aucuparia & - & - & - & - & - & - & - & II & III & $\mathrm{I}$ & III & - \\
\hline Cotoneaster matrensis & - & - & - & - & - & - & - & - & $\mathrm{I}$ & - & - & - \\
\hline Rosa pendulina & - & - & - & - & - & - & II & - & $\mathrm{V}$ & - & I & - \\
\hline Rosa spinosissima & - & - & - & - & - & - & - & - & I & - & - & - \\
\hline Rubus idaeus & - & - & - & - & - & I & - & II & $\mathrm{V}$ & II & III & II \\
\hline Sambucus racen & - & - & - & - & - & - & - & $\mathrm{I}$ & IV & - & I & - \\
\hline Spiraea media & - & - & - & - & - & - & I & - & I & - & - & - \\
\hline Asplenium septentrionale & - & - & - & - & - & - & - & $\mathrm{I}$ & III & - & - & - \\
\hline Chrysosplenium alternifolium & - & - & - & - & - & - & - & - & I & - & - & - \\
\hline Dryopteris carthusiana s. str. & - & - & - & - & - & - & - & $\mathrm{I}$ & I & - & - & - \\
\hline Dryopteris dilatata & - & - & - & - & - & - & - & - & $\mathrm{I}$ & - & - & - \\
\hline Festuca altissima & - & - & - & - & - & - & - & - & I & - & - & - \\
\hline Luzula luzuloides & - & - & - & - & - & - & I & - & II & - & - & - \\
\hline Sedum acre & - & - & - & - & - & - & - & - & I & - & - & - \\
\hline Solanum dulcamara & - & - & - & - & - & I & - & I & III & - & - & - \\
\hline Viscaria vulgaris & - & - & - & - & - & - & - & $\mathrm{I}$ & $\mathrm{I}$ & - & - & - \\
\hline \multicolumn{13}{|c|}{$\begin{array}{l}\text { 1: Keszthely Mts (Kevey ined.), 2: Bakony Mts (Kevey ined.), 3: Vértes Mts (Isépy 1968), 4: } \\
\text { Gerecse Mts (Kevey ined.), 5: Buda Mts (Zólyomi 1958), 6: Börzsöny Mts (Nagy ined.), 7: } \\
\text { Mátra Mts (Kovács 1968), 8: Zemplén Mts (Simon 1977), 9: Cerová Vrchovina (Csiky ined.), } \\
\text { 10: Swiss Jura (Oberdorfer 1957), 11: Frankenwald (Oberdorfer 1957), 12: Schwarzwald } \\
\text { (Oberdorfer 1957) }\end{array}$} \\
\hline
\end{tabular}

species of the Mercuriali-Tilietum are already absent in the northest part of the Northern Hungarian Mts (Zemplén Mts).

The majority of the positive differential species (represented in the Roso pendulinae-Tilietum cordatae with values of at least two frequency categories higher than in other associations) belong mainly to the "gap species" of the Carpathian beech forests (e.g. Sorbus aucuparia, Sambucus racemosa, Rosa pendulina, Rubus idaeus, Populus tremula, Betula pendula, Dryopteris dilatata, etc.), and may be considered as relics of the subboreal phase. Xerophilous grassland plants, such as Asplenium septentrionale, Sedum acre, Spiraea media, Cotoneaster matrensis, acidophilous forest elements (e.g. Luzula luzuloides, Viscaria vulgaris) and alder wood species (e. g. Dryopteris carthusiana, Chrysosplenium alternifolium, Solanum dulcamara) are also typical of this block forest, but occurring less frequently. 
Table 4

Roso pendulinae-Tilietum cordatae

Phragmitetea

Solanum dulcamara (Cal, Bia, Spu, Ate, AP) $\begin{array}{llllllll}1 & 2 & 3 & 4 & 5 & 6 & 7 & 8\end{array}$

Arrhenatheretea incl. Arrhenatheretalia)

Anthriscus sylvestris (Ar, GA, Spu, AP)

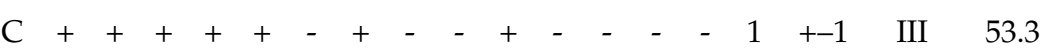

Calluno-Ulicetea (incl. Vaccinio-Genistetalia et Calluno-Genistion)

Betula pendula (Qrp, AbP)

Genistion)

Festuco-Brom acre

Festucetalia valesiacae

Cardaminopsis arenosa (TAc, Qpp)

Allium montanum

Asplenio-Festucion pallentis

Polypodium vulgare (TAc, Qrp)

Asplenium septentrionale

Asplenium trichomanes (BrF, TAc, Qrp, OCn)

Calystegion sepium

Myosoton aquaticum (Pte, Spu, Ate, AP)

Epilobietea angustifolii (incl. Epilobietalia)

Rubus idaeus (US, CF)

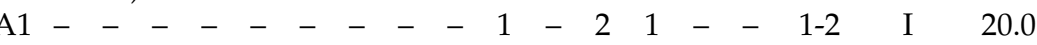

B1 - + + + - + $+++-+1+-+-1$ III 60.0

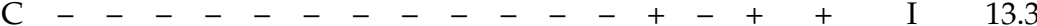

$\mathrm{S}-+++-\ldots+-+1-22+++-2 \quad$ IV 66.7

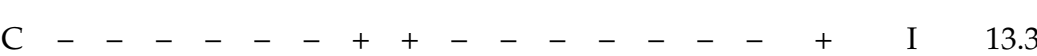

C $-++++++-\ldots+-+++-+$ III 53.3

$\mathrm{C}-\ldots-\ldots-+\ldots-\ldots+\ldots$ I 6.7

$\begin{array}{lllllllllllllllllll}\mathrm{C} & 2 & 2 & 1 & 2 & 1 & 1 & - & + & 1 & 1 & 2 & 2 & + & 1 & 2 & +-2 & \mathrm{~V} & 93.3\end{array}$

C $-\ldots++-++++\ldots++1++-1$ III 53.3

$\mathrm{C}+++-\ldots+-\ldots+-+-\ldots+$ II 33.3

C $-+\ldots+\ldots+\ldots+\ldots$ I 6.7

B1 $111411+++11+1+1++-1$ V 100.0

$\mathrm{C}+1++++++++-+++-+-1 \quad \mathrm{~V} \quad 86.7$

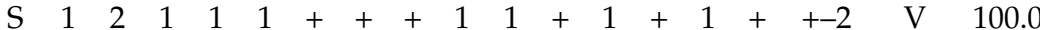

Salix caprea (US, QF)

A1 1 _

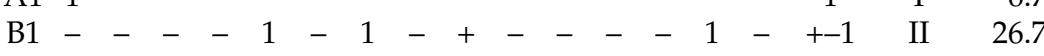

S $1-5-\begin{array}{lllllllllllllll} & 1 & - & 1 & - & + & - & - & - & 1 & - & +-1 & \text { II } & 33.3\end{array}$

Epilobium angustifolium (Epn, Qrp)

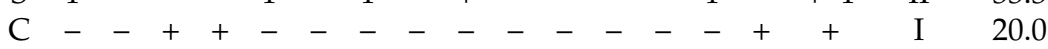




\begin{tabular}{|c|c|c|c|c|c|c|c|c|c|c|c|c|c|c|c|c|c|c|c|c|}
\hline \multicolumn{20}{|c|}{ Table 4 (cont.) } & \\
\hline & & 1 & 2 & 3 & 4 & 5 & 6 & 7 & 8 & 9 & 10 & 11 & 12 & 13 & 14 & 15 & A-D & $\mathrm{K}$ & $\%$ & \\
\hline \multicolumn{20}{|c|}{ Urtico-Sambucetea (incl. Sambucetalia et Sambuco-Salicion capreae) } & \\
\hline \multirow[t]{3}{*}{ Sambucus racemosa (EuF, TAc) } & B1 & 1 & 1 & 1 & 1 & 2 & 1 & + & 1 & - & 1 & - & - & 1 & - & + & +-2 & IV & 73.3 & \\
\hline & $\mathrm{C}$ & + & + & + & + & + & + & + & + & - & + & - & - & - & + & + & + & IV & 73.3 & T \\
\hline & $\mathrm{S}$ & 1 & 1 & 1 & 1 & 2 & 1 & + & 1 & - & 1 & - & - & 1 & + & + & +-2 & IV & 80.0 & \\
\hline \multicolumn{20}{|c|}{ 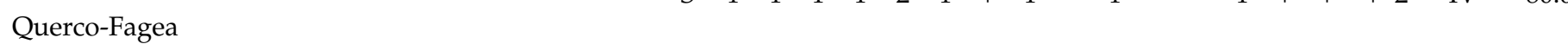 } & \\
\hline \multirow[t]{3}{*}{ Euonymus verrucosus (Qpp, Pru) } & B1 & + & 1 & 1 & 1 & 1 & 1 & 1 & 1 & 1 & 1 & 1 & 1 & 1 & 1 & 1 & +-1 & $\mathrm{~V}$ & 100.0 & $=$ \\
\hline & $\mathrm{C}$ & + & + & + & + & + & + & + & + & + & + & 1 & + & + & + & 1 & +-1 & $\mathrm{~V}$ & 100.0 & \\
\hline & $\mathrm{S}$ & + & 1 & 1 & 1 & 1 & 1 & 1 & 1 & 1 & 1 & 2 & 1 & 1 & 1 & 2 & +-2 & $\mathrm{~V}$ & 100.0 & \\
\hline Poa nemoralis & $\mathrm{C}$ & + & 1 & + & 1 & + & + & 1 & 1 & 1 & 1 & + & 1 & 1 & 1 & 1 & +-1 & $\mathrm{~V}$ & 100.0 & \\
\hline Sedum maximum (FB, TAc, Qpp) & $\mathrm{C}$ & + & 1 & 1 & 1 & + & + & + & + & + & + & + & 1 & + & + & + & +-1 & $\mathrm{~V}$ & 100.0 & \\
\hline Geranium robertianum (Epa, CF) & $\mathrm{C}$ & 1 & 1 & 1 & 1 & 1 & 1 & 1 & 1 & 1 & 1 & 1 & 1 & + & - & + & +-1 & $\mathrm{~V}$ & 93.3 & \\
\hline Mycelis muralis & $\mathrm{C}$ & + & + & + & + & - & + & + & - & + & + & + & + & + & + & + & + & $\mathrm{V}$ & 86.7 & \\
\hline \multirow[t]{4}{*}{ Corylus avellana } & A1 & 1 & - & - & - & - & - & 1 & - & - & 1 & - & - & - & - & - & 1 & I & 20.0 & \\
\hline & B1 & + & - & 1 & 1 & 1 & 1 & 1 & + & - & 1 & 1 & 1 & 1 & 1 & - & +-1 & IV & 80.0 & \\
\hline & $\mathrm{C}$ & + & - & - & + & - & - & - & - & - & - & + & + & + & - & - & + & II & 33.3 & \\
\hline & $S$ & 1 & - & 1 & 1 & 1 & 1 & 2 & + & - & 2 & 1 & 1 & 1 & 1 & - & +-2 & IV & 80.0 & \\
\hline \multirow[t]{4}{*}{ Quercus petraea agg. (Cp, Qrp, Qpp) } & A1 & - & - & - & 1 & 1 & 1 & 2 & 1 & - & 1 & 1 & - & 2 & 3 & + & +-3 & IV & 66.7 & \\
\hline & B1 & - & - & - & - & - & - & + & - & - & - & - & + & - & - & - & + & $\mathrm{I}$ & 13.3 & \\
\hline & $\mathrm{C}$ & - & - & - & - & - & - & + & - & - & + & - & - & + & + & - & + & II & 26.7 & \\
\hline & $\mathrm{S}$ & - & - & - & 1 & 1 & 1 & 2 & 1 & - & 1 & 1 & + & 2 & 3 & + & +-3 & IV & 73.3 & \\
\hline Fallopia dumetorum (GA) & $\mathrm{C}$ & - & + & - & - & + & + & + & - & - & + & + & + & + & + & + & + & IV & 66.7 & \\
\hline Galium schultesii (Cp, Qpp) & $\mathrm{C}$ & - & + & - & + & + & - & + & - & - & 1 & - & + & 1 & 1 & + & +-1 & III & 60.0 & \\
\hline \multirow[t]{4}{*}{ Populus tremula (Qrp, Qia) } & A1 & - & 2 & 1 & 1 & - & - & - & - & - & - & 1 & - & 1 & 1 & 2 & $1-2$ & III & 46.7 & \\
\hline & B1 & - & + & - & - & - & - & + & 1 & - & - & 1 & - & 1 & 1 & + & +-1 & III & 46.7 & \\
\hline & $\mathrm{C}$ & - & - & - & - & - & - & - & - & - & - & - & - & - & - & + & + & $\mathrm{I}$ & 6.7 & \\
\hline & $\mathrm{S}$ & - & 2 & 1 & 1 & - & - & + & 1 & - & - & 2 & - & 2 & 2 & 2 & +-2 & III & 60.0 & \\
\hline Fragaria vesca (Epa) & $\mathrm{C}$ & + & - & - & + & - & - & - & + & - & + & - & + & + & + & + & + & III & 53.3 & \\
\hline \multirow[t]{2}{*}{ Acer campestre } & A1 & - & - & - & - & 2 & 1 & 2 & 1 & - & - & - & - & 1 & - & - & $1-2$ & II & 33.3 & \\
\hline & B1 & - & - & - & - & 1 & + & 1 & + & - & - & - & - & 1 & 1 & - & +-1 & II & 40.0 & \\
\hline
\end{tabular}


Table 4 (cont.)

\begin{tabular}{|c|c|c|c|c|c|c|c|c|c|c|c|c|c|c|c|c|c|c|c|}
\hline & & 1 & 2 & 3 & 4 & 5 & 6 & 7 & 8 & 91 & 10 & 11 & 121 & 13 & 141 & 15 & A-D & $\mathrm{K}$ & $\%$ \\
\hline \multirow[t]{2}{*}{ Acer campestre } & $\mathrm{C}$ & - & - & - & - & + & + & + & + & - & - & - & - & + & + & + & + & III & 46.7 \\
\hline & $S$ & - & - & - & - & 2 & 1 & 2 & 1 & - & - & - & - & 2 & 1 & + & +-2 & III & 46.7 \\
\hline Campanula rapunculoides (Epa) & $\mathrm{C}$ & + & - & - & + & - & - & - & - & - & + & - & + & + & + & + & + & III & 46.7 \\
\hline \multirow[t]{4}{*}{ Tilia cordata (Cp, Qpp) } & A1 & 3 & 2 & - & 2 & 3 & - & - & 2 & - & 2 & - & - & - & - & - & $2-3$ & II & 40.0 \\
\hline & B1 & 1 & 1 & - & 1 & + & - & - & 1 & - & 1 & - & - & - & - & - & +-1 & II & 40.0 \\
\hline & $\mathrm{C}$ & + & + & + & + & + & - & - & + & - & + & - & - & - & - & - & + & III & 46.7 \\
\hline & $S$ & 3 & 2 & + & 2 & 3 & - & - & 2 & - & 2 & - & - & - & - & - & +-3 & III & 46.7 \\
\hline Veronica chamaedrys subsp. vindobonensis (Ara) & $\mathrm{C}$ & - & - & - & - & - & - & + & + & + & + & - & 1 & 1 & + & - & +-1 & III & 46.7 \\
\hline \multirow[t]{3}{*}{ Cornus sanguinea (Qpp) } & B1 & - & - & - & + & + & + & - & - & 1 & 1 & - & - & - & - & - & +-1 & II & 33.3 \\
\hline & $\mathrm{C}$ & - & - & - & - & - & - & - & - & - & - & - & - & + & - & - & + & I & 6.7 \\
\hline & $\mathrm{S}$ & - & - & - & + & + & + & - & - & 1 & 1 & - & - & + & - & - & +-1 & II & 40.0 \\
\hline Pulmonaria obscura (CF) & $\mathrm{C}$ & - & + & - & + & + & + & - & - & - & 1 & - & - & - & - & + & +-1 & II & 40.0 \\
\hline Campanula persicifolia & $\mathrm{C}$ & - & - & - & + & - & - & + & - & - & - & - & - & + & + & + & + & II & 33.3 \\
\hline \multirow[t]{3}{*}{ Lonicera xylosteum } & B1 & 1 & 2 & 1 & + & - & - & - & - & - & - & - & - & - & - & - & +-2 & II & 26.7 \\
\hline & $\mathrm{C}$ & - & + & + & + & - & - & - & - & - & - & - & - & - & - & + & + & II & 26.7 \\
\hline & S & 1 & 2 & 1 & + & - & - & - & - & - & - & - & - & - & - & + & +-2 & II & 33.3 \\
\hline \multirow[t]{3}{*}{ Rhamnus catharticus (Qpp, Pru) } & B1 & - & - & - & - & 1 & - & 1 & - & - & 1 & - & - & - & - & - & 1 & I & 20.0 \\
\hline & $\mathrm{C}$ & - & - & - & - & - & - & - & - & - & - & - & - & + & - & + & + & I & 13.3 \\
\hline & S & - & - & - & - & 1 & - & 1 & - & - & 1 & - & - & + & - & + & +-1 & II & 33.3 \\
\hline Stellaria holostea (CF, Cp) & $\mathrm{C}$ & - & - & - & - & - & + & + & - & - & 1 & - & + & - & - & + & +-1 & II & 33.3 \\
\hline Cruciata glabra & $\mathrm{C}$ & - & - & - & - & - & - & + & - & - & + & - & + & - & + & - & + & II & 26.7 \\
\hline Convallaria majalis & $\mathrm{C}$ & - & - & - & 1 & - & - & - & - & - & - & - & - & 1 & - & + & +-1 & I & 20.0 \\
\hline Euonymus europaea (Qpp) & B1 & - & - & - & - & 1 & - & - & - & + & + & - & - & - & - & - & +-1 & I & 20.0 \\
\hline Galeopsis pubescens (Epa) & $\mathrm{C}$ & - & - & - & + & - & - & - & - & + & - & - & - & - & + & - & + & I & 20.0 \\
\hline Carex divulsa (CF) & $\mathrm{C}$ & - & - & - & - & - & - & - & + & - & - & - & - & - & + & - & + & I & 13.3 \\
\hline Clinopodium vulgare (Qpp) & $\mathrm{C}$ & - & - & - & - & - & - & + & + & - & - & - & - & - & - & - & + & I & 13.3 \\
\hline Dactylis polygama $(\mathrm{Cp})$ & $\mathrm{C}$ & - & - & - & - & - & - & + & - & - & - & - & - & - & + & - & + & I & 13.3 \\
\hline Heracleum sphondylium (MoA) & $\mathrm{C}$ & - & - & - & - & - & - & - & - & - & + & - & - & + & - & - & + & I & 13.3 \\
\hline Ligustrum vulgare (Cp, Qpp) & B1 & - & - & - & - & - & - & - & - & - & + & - & - & + & - & - & + & I & 13.3 \\
\hline Melica nutans & $\mathrm{C}$ & - & - & - & - & - & - & - & - & - & - & - & - & + & + & - & + & $\mathrm{I}$ & 13.3 \\
\hline
\end{tabular}


Table 4 (cont.)

\begin{tabular}{|c|c|c|c|c|c|c|c|c|c|c|c|c|c|c|c|c|c|c|c|}
\hline & & 1 & 2 & 3 & 4 & 5 & 6 & 7 & 8 & 9 & 10 & 11 & 12 & 13 & 14 & 15 & A-D & $\mathrm{K}$ & $\%$ \\
\hline Ajuga reptans (MoA) & $\mathrm{C}$ & - & - & - & - & - & - & - & - & - & - & - & - & - & + & - & + & I & 6.7 \\
\hline Campanula trachelium (Epa, Cp) & $\mathrm{C}$ & - & - & + & - & - & - & - & - & - & - & - & - & - & - & - & + & I & 6.7 \\
\hline Clematis vitalba & $\mathrm{C}$ & - & - & - & - & - & - & - & + & - & - & - & - & - & - & - & + & I & 6.7 \\
\hline Hieracium sabaudum agg. (Qrp, AbP) & $\mathrm{C}$ & - & - & - & - & - & - & - & - & - & - & - & - & + & - & - & + & I & 6.7 \\
\hline Hieracium sylvaticum agg. (Epa, Qrp) & $\mathrm{C}$ & - & - & - & - & - & - & - & - & - & - & - & - & - & - & + & + & I & 6.7 \\
\hline Hypericum montanum & $\mathrm{C}$ & - & - & - & - & - & - & - & - & - & - & - & - & + & - & - & + & I & 6.7 \\
\hline Melica uniflora $(\mathrm{Cp})$ & $\mathrm{C}$ & - & - & - & - & - & - & - & - & - & - & - & - & - & - & + & + & I & 6.7 \\
\hline Scrophularia nodosa (GA, Epa) & $\mathrm{C}$ & - & - & - & - & - & - & - & - & - & - & - & - & + & - & - & + & I & 6.7 \\
\hline $\begin{array}{l}\text { Symphytum tuberosum subsp. angustifolium (CF, Cp) } \\
\text { Salicion albae }\end{array}$ & $\mathrm{C}$ & - & - & - & - & - & - & - & - & - & - & - & - & - & - & + & + & I & 6.7 \\
\hline \multirow[t]{3}{*}{ Humulus lupulus (Cal, Ate, AP) } & B1 & - & - & - & - & - & - & - & - & - & - & - & - & + & - & - & + & $\mathrm{I}$ & 6.7 \\
\hline & C & - & - & - & - & + & - & 1 & + & - & - & - & - & + & + & - & +-1 & II & 33.3 \\
\hline & $\mathrm{S}$ & - & - & - & - & + & - & 1 & + & - & - & - & - & + & + & - & +-1 & II & 33.3 \\
\hline \multicolumn{20}{|l|}{ Alnetea glutinosae } \\
\hline Dryopteris carthusiana s.str. (CF, Agi, Qrp, VP) & $\mathrm{C}$ & 1 & - & + & - & - & - & - & - & - & - & - & - & - & - & - & +-1 & I & 13.3 \\
\hline Dryopteris dilatata (CF, Agi, Qrp, VP) & $\mathrm{C}$ & + & - & - & - & - & - & - & - & - & - & - & - & - & - & + & + & I & 13.3 \\
\hline \multicolumn{20}{|l|}{ Carpino-Fagetea (incl. Fagetalia) } \\
\hline Dryopteris filix-mas s. str. & $\mathrm{C}$ & 2 & 2 & 2 & 2 & 1 & 1 & 1 & 1 & 2 & 2 & 1 & 1 & 1 & 1 & 2 & $1-2$ & $\mathrm{~V}$ & 100.0 \\
\hline \multirow[t]{4}{*}{ Carpinus betulus (Cp) } & A1 & - & - & - & - & 2 & 2 & 1 & 2 & 2 & 1 & - & - & - & 2 & 3 & $1-3$ & III & 53.3 \\
\hline & B1 & - & + & - & - & + & 1 & + & 1 & 1 & 1 & - & + & - & + & 1 & +-1 & IV & 66.7 \\
\hline & $\mathrm{C}$ & - & - & - & - & + & + & + & + & + & + & - & - & - & + & + & + & III & 53.3 \\
\hline & $\mathrm{S}$ & - & + & - & - & 2 & 2 & 1 & 2 & 2 & 2 & - & + & - & 2 & 3 & +-3 & IV & 66.7 \\
\hline \multirow[t]{4}{*}{ Fagus sylvatica (EuF) } & A1 & - & 2 & - & - & - & - & 1 & - & 1 & - & 1 & 3 & 3 & 2 & 1 & $1-3$ & III & 53.3 \\
\hline & B1 & - & 1 & - & - & - & - & - & - & + & - & - & 1 & 1 & - & 1 & +-1 & II & 33.3 \\
\hline & $\mathrm{C}$ & - & + & - & - & - & - & + & - & - & - & - & + & + & + & - & + & II & 33.3 \\
\hline & $\mathrm{S}$ & - & 2 & - & - & - & - & 1 & - & 1 & - & 1 & 3 & 3 & 2 & 2 & $1-3$ & III & 53.3 \\
\hline \multirow[t]{4}{*}{ Sorbus aucuparia (Qrp, Qpp, VP) } & A1 & 2 & 2 & 2 & 2 & - & - & - & - & - & 2 & 1 & - & 1 & 1 & - & $1-2$ & III & 53.3 \\
\hline & B1 & 1 & 1 & 1 & + & - & - & - & - & - & 1 & 1 & - & 1 & + & - & +-1 & III & 53.3 \\
\hline & $\mathrm{C}$ & + & + & + & + & - & - & - & - & - & + & + & - & + & + & - & + & III & 53.3 \\
\hline & $\mathrm{S}$ & 2 & 2 & 2 & 2 & - & - & - & - & - & 2 & 2 & - & 2 & 1 & - & $1-2$ & III & 53.3 \\
\hline
\end{tabular}




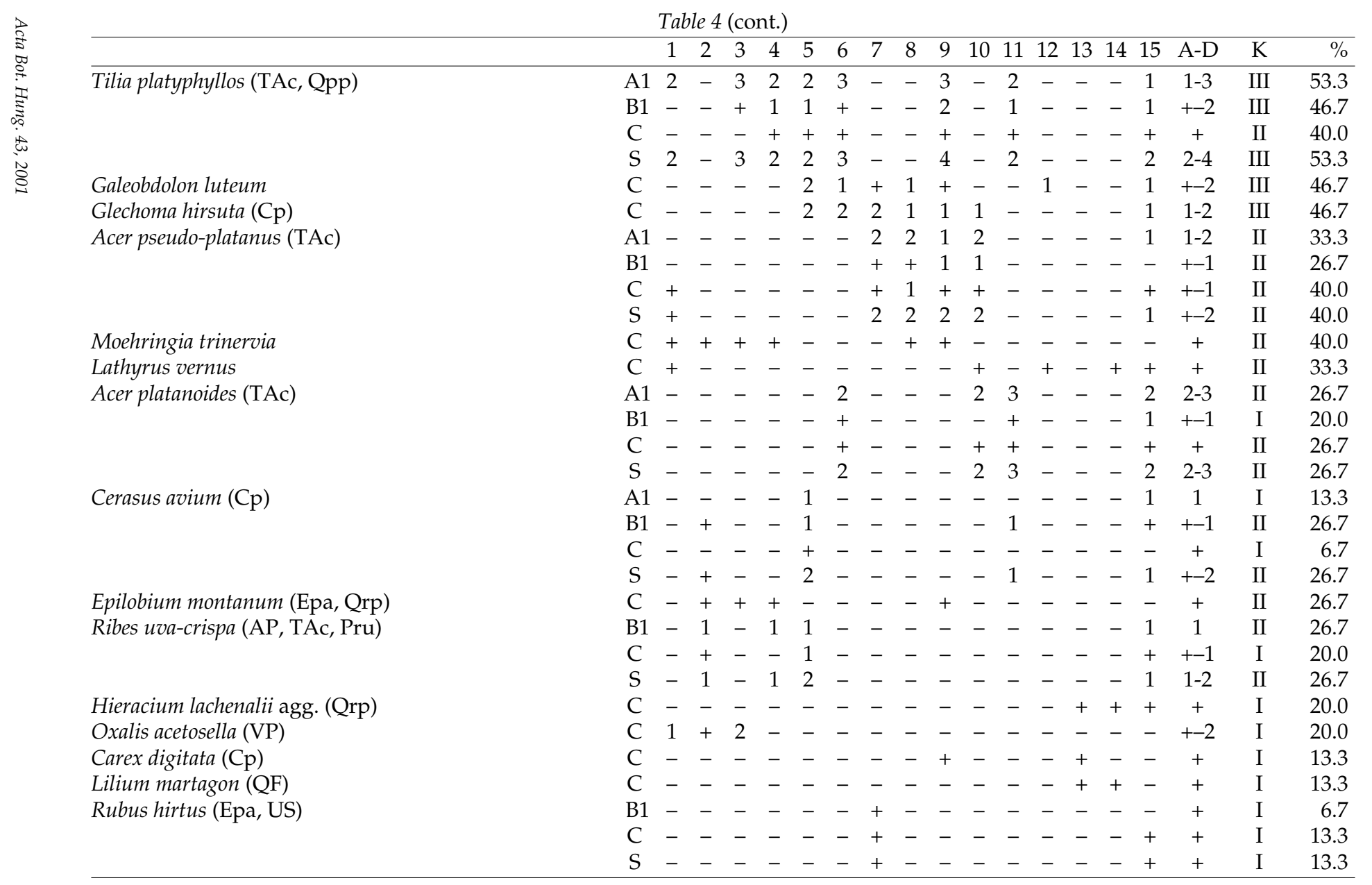


Table 4 (cont.)

\begin{tabular}{|c|c|c|c|c|c|c|c|c|c|c|c|c|c|c|c|c|c|c|c|}
\hline & & 1 & 2 & 3 & 4 & 5 & 6 & 7 & 8 & 9 & 10 & 11 & 12 & 13 & 14 & 15 & A-D & $\mathrm{K}$ & $\%$ \\
\hline Viola sylvestris & $\mathrm{C}$ & + & - & - & - & - & - & - & + & - & - & - & - & - & - & - & + & $\mathrm{I}$ & 13.3 \\
\hline Actaea spicata (EuF, TAc) & C & - & - & - & - & - & - & + & - & - & - & - & - & - & - & - & + & $\mathrm{I}$ & 6.7 \\
\hline Athyrium filix-femina (Qrp, VP) & $\mathrm{C}$ & + & - & - & - & - & - & - & - & - & - & - & - & - & - & - & + & I & 6.7 \\
\hline \multirow[t]{3}{*}{ Hedera helix } & A1 & - & - & - & - & - & + & - & - & - & - & - & - & - & - & - & + & I & 6.7 \\
\hline & $\mathrm{C}$ & - & - & - & - & - & + & - & - & - & - & - & - & - & - & - & + & I & 6.7 \\
\hline & $S$ & - & - & - & - & - & + & - & - & - & - & - & - & - & - & - & + & I & 6.7 \\
\hline Salvia glutinosa & $\mathrm{C}$ & - & - & - & - & - & + & - & - & - & - & - & - & - & - & - & + & $\mathrm{I}$ & 6.7 \\
\hline \multirow[t]{4}{*}{ Ulmus glabra (TAc) } & A1 & - & - & - & - & - & 1 & - & - & - & - & - & - & - & - & - & 1 & I & 6.7 \\
\hline & B1 & - & - & - & - & - & + & - & - & - & - & - & - & - & - & - & + & I & 6.7 \\
\hline & C & - & - & - & - & - & + & - & - & - & - & - & - & - & - & - & + & I & 6.7 \\
\hline & $\mathrm{S}$ & - & - & - & - & - & 1 & - & - & - & - & - & - & - & - & - & 1 & I & 6.7 \\
\hline \multicolumn{20}{|l|}{ Alno-Padion } \\
\hline Impatiens noli-tangere (Sal) & C & + & + & 1 & + & - & - & - & - & - & - & - & - & - & - & - & +-1 & II & 26.7 \\
\hline Chrysosplenium alternifolium (TAc) & C & - & - & 1 & - & - & - & - & - & - & - & - & - & - & - & - & 1 & I & 6.7 \\
\hline Festuca gigantea (Cal, Epa) & C & - & - & - & - & - & - & - & + & - & - & - & - & - & - & - & + & I & 6.7 \\
\hline \multirow{4}{*}{$\begin{array}{l}\text { Eu-Fagion } \\
\text { Rosa pendulina }\end{array}$} & & & & & & & & & & & & & & & & & & & \\
\hline & B1 & 1 & 1 & 1 & - & 2 & - & 1 & 1 & 1 & 2 & 2 & 2 & 1 & + & + & +-2 & $\mathrm{~V}$ & 86.7 \\
\hline & $\mathrm{C}$ & + & + & + & - & + & - & + & 1 & + & 1 & 1 & + & 1 & + & + & +-1 & $\mathrm{~V}$ & 86.7 \\
\hline & $\mathrm{S}$ & 1 & 1 & 1 & - & 2 & - & 1 & 2 & 1 & 2 & 2 & 2 & 2 & + & + & +-2 & $\mathrm{~V}$ & 86.7 \\
\hline Festuca altissima (TAc) & $\mathrm{C}$ & - & - & - & - & - & - & - & - & - & - & - & - & 2 & - & - & 2 & I & 6.7 \\
\hline Tilio-Acerion & & & & & & & & & & & & & & & & & & & \\
\hline Cystopteris fragilis (AFe) & C & + & + & + & + & + & + & + & + & + & + & + & 1 & - & + & + & +-1 & $\mathrm{~V}$ & 93.3 \\
\hline Quercetea robori-petraeae (incl. Pino-Quercetalia) & & & & & & & & & & & & & & & & & & & \\
\hline Luzula luzuloides (CU, AbP) & C & - & - & + & + & - & - & - & - & - & - & - & 1 & - & 1 & + & +-1 & II & 33.3 \\
\hline Calamagrostis arundinacea (Еpa) & $\mathrm{C}$ & - & - & - & - & - & - & - & - & - & - & - & - & 1 & 2 & - & $1-2$ & I & 13.3 \\
\hline Viscaria vulgaris (Qpp) & $\mathrm{C}$ & - & - & - & - & - & - & - & + & - & - & - & + & - & - & - & + & I & 13.3 \\
\hline Hieracium umbellatum agg. (NC, Epa, Qpp, PP) & $\mathrm{C}$ & - & - & - & - & - & - & - & - & - & - & - & - & + & - & - & + & I & 6.7 \\
\hline Veronica officinalis (NC, Epa, PP, VP) & $\mathrm{C}$ & - & - & - & - & - & - & - & - & - & - & + & - & - & - & - & + & $\mathrm{I}$ & 6.7 \\
\hline
\end{tabular}


Table 4 (cont.)

Polygonatum odoratum $(\mathrm{Fvl})$

Chamaecytisus hirsutus (Fvl)

Rosa spinosissima (Pru)

$\mathrm{B} 1-1-+1+1+++1-1++++1$ IV $\quad 80.0$

$\mathrm{C}-\ldots++++++++\ldots++\ldots$ III 53.3

$\mathrm{S}-1-1+1+1+++1-1+++-1$ IV -80.0

C - _ _ _ _ _ _ _ _ _

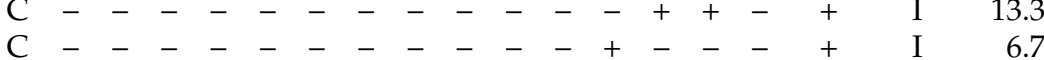

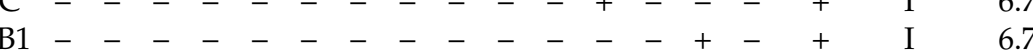

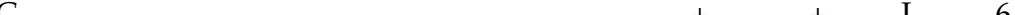

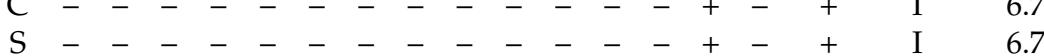

$\begin{array}{llllllllllllllllll}\mathrm{S}- & - & - & - & - & - & - & - & - & - & + & - & + & \mathrm{I} & 6.7 \\ \mathrm{C}- & - & - & - & - & - & - & - & - & - & - & + & - & + & \mathrm{I} & 6.7\end{array}$

Solidago virga-aurea (NC, Epa, Qrp)

Vincetoxicum hirundinaria $(\mathrm{Fvl})$

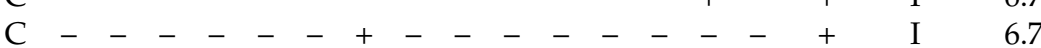

Orno-Cotinion

Sorbus graeca

Quercetalia pubescentis-petraeae

Valeriana stolonifera

Trifolium medium

Aceri tatarico-Quercion

Spiraea media $(\mathrm{SFe}, \mathrm{AFe})$

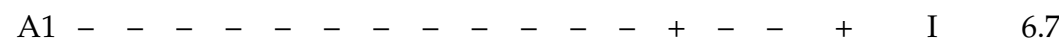

$\mathrm{C}-{ }_{-}-{ }_{-}-{ }_{-}+{ }_{-}++-+$II 26.7

C $-\ldots-\ldots-\ldots-\ldots-\ldots+\ldots$ I 6.7

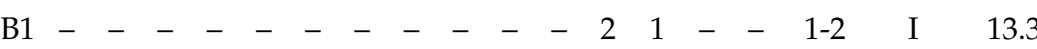

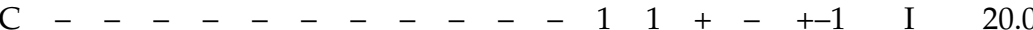

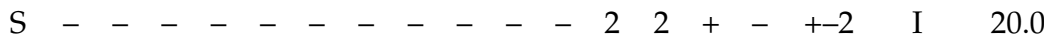

Cotoneaster matrensis (Qpp)

Indifferent

Urtica dioica (Ar, GA, Epa, Spu)

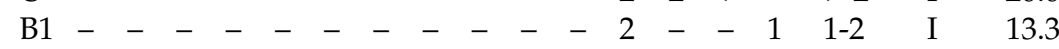

Taraxacum officinale (MoA, FPe, CyF, ChS)

Galium aparine (Sea, Epa, QF)

Chelidonium majus (Che, Ar, GA, Epa)

Euphorbia cyparissias (FB, ChS, Epa, Qpp)

Hypericum perforatum (NC, FB, Qpp, PP)

Poa angustifolia (Ara, FPi, FBt, ChS, Qpp)

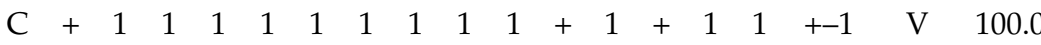

$\mathrm{C}+++++++++--++-++$ IV 80.0

$\mathrm{C}-+-+++1++++--++++1$ IV 73.3

$\mathrm{C}-\ldots++1+-+\ldots-\ldots+\ldots++-1$ II 40.0

$\mathrm{C}-\mathrm{C}_{-}-\mathrm{C}_{-}+\mathrm{C}_{-}+\mathrm{C}_{-}+\mathrm{I}$ I 13.3

$\mathrm{C}-\mathrm{C}_{-}-++\ldots-\ldots+\ldots$ I 13.3

$\begin{array}{llllllllllllllllll}C & - & - & - & - & - & + & + & - & - & - & - & - & - & - & + & \mathrm{I} & 13.3 \\ \mathrm{C}- & - & - & - & - & - & - & + & + & - & - & - & - & - & - & + & \mathrm{I} & 13.3\end{array}$

Galium mollugo (MoA, FBt, Qrp, Qpp) 
Table 4 (cont.)

\begin{tabular}{|c|c|c|c|c|c|c|c|c|c|c|c|c|c|c|c|c|c|c|c|}
\hline & & 1 & 2 & 3 & 4 & 5 & 6 & 7 & 8 & 9 & 10 & 11 & 12 & 13 & 14 & 15 & A-D & $\mathrm{K}$ & $\%$ \\
\hline Juniperus communis (NC, Fvg, Qpp, EP, PP) & B1 & - & - & - & - & - & - & - & - & - & - & - & - & - & + & - & + & $\mathrm{I}$ & 6.7 \\
\hline Silene vulgaris (Ara, Fvl, Qpp) & $\mathrm{C}$ & - & - & - & - & + & - & - & - & - & - & - & - & - & - & - & + & I & 6.7 \\
\hline
\end{tabular}

Roso pendulinae-Tilietum cordatae (Basic data of relevés)

\begin{tabular}{lccccccccccccccc}
\hline Localities & 1. & 2. & 3. & 4. & 5. & 6. & 7. & 8. & 9. & 10. & 11. & 12. & 13. & 14. & 15. \\
\hline Serial number & 2394 & 2395 & 2396 & 2397 & 2398 & 2399 & 2400 & 2401 & 2402 & 2403 & 2404 & 2405 & 2369 & 2370 & 2371 \\
Year & 1999 & 1999 & 1999 & 1999 & 1999 & 1999 & 1999 & 1999 & 1999 & 1999 & 1999 & 1999 & 1999 & 1999 & 1999 \\
Date of relevé & 20.06 & 20.06 & 20.06 & 20.06 & 20.06 & 20.06 & 21.06 & 21.06 & 21.06 & 21.06 & 12.07 & 12.07 & 17.08 & 17.08 & 17.08 \\
Basic rock & basalt & basalt & basalt & basalt & basalt & basalt & basalt & basalt & basalt & basaltandesitebasalt & basalt & basalt & basalt \\
Block surface, mean (m×m) & $1 \times 2$ & $1 \times 2$ & $1 \times 2$ & $1 \times 1$ & $0.5 \times 1$ & $0.5 \times 1$ & $0.5 \times 1$ & $0.5 \times 1$ & $1 \times 1$ & $0.5 \times .5$ & $0.3 \times .5$ & $0.2 \times .3$ & $1 \times 2$ & $1 \times 2$ & $1 \times 1$ \\
Altitude (m asl.) & 500 & 500 & 510 & 520 & 500 & 500 & 540 & 500 & 460 & 500 & 500 & 450 & 530 & 525 & 530 \\
Exposition (degree) & 360 & 345 & 330 & 270 & 260 & 285 & 270 & 285 & 270 & 315 & 40 & 5 & 195 & 210 & 60 \\
Declination (degree) & 25 & 25 & 35 & 30 & 25 & 25 & 35 & 35 & 20 & 10 & 35 & 20 & 10 & 15 & 60 \\
Upper canopy, cover (\%) & 60 & 60 & 55 & 60 & 60 & 60 & 55 & 50 & 65 & 60 & 50 & 60 & 60 & 50 & 50 \\
Shrub layer, cover (\%) & 15 & 35 & 25 & 20 & 30 & 10 & 10 & 10 & 15 & 25 & 35 & 35 & 35 & 15 & 10 \\
Herb layer, cover (\%) & 25 & 30 & 40 & 25 & 35 & 30 & 20 & 15 & 15 & 50 & 20 & 30 & 35 & 25 & 25 \\
Moss layer, cover (\%) & 90 & 70 & 80 & 70 & 60 & 40 & 20 & 50 & 70 & 40 & 50 & 40 & 50 & 40 & 40 \\
Upper canopy, height (m) & 15 & 15 & 18 & 18 & 17 & 18 & 17 & 18 & 18 & 15 & 20 & 20 & 17 & 15 & 10 \\
Shrub layer, height (m) & 5 & 5 & 5 & 5 & 5 & 5 & 3 & 5 & 5 & 5 & 5 & 4 & 5 & 5 & 3 \\
Plot size (m ${ }^{2}$ ) & 400 & 400 & 400 & 400 & 400 & 400 & 400 & 400 & 400 & 400 & 400 & 400 & 400 & 400 & 400 \\
\hline
\end{tabular}

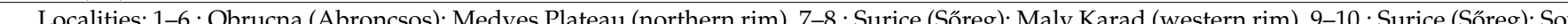
(Egyházasbást): Pohansky Vrch (southern rim), 15: Stan 2394). Relevés made by Csiky, J. (ined.).

Abbreviations in the phytosociological tables. A1: upper canopy, AbP: Abieti-Piceea, AFe: Asplenio-Festucion pallentis, Agi: Alnion glutinosae-incanae, AP: Alno-Padion, Ar: Artemisietea, Ara: Arrhenatheretea, Ate: Alnetea glutinosae, B1: shrub layer, Bia: Bidentetea, BrF: Bromo-Festucion pallentis, C: herb layer, Cal: Calystegion sepium, CF: Carpino-Fagetea, Che: Chenopodietea, ChS: Chenopodio-Scleranthea, Cp: Carpinion, CU: Calluno-Ulicetea, CyF: Cynodonto-Festucion, EP: Erico-Pinetea, Epa: Epilobietea angustifolii, Epn: Epilobion angustifolii, EuF: Eu-Fagion, FB: Festuco-Bromea, FBt: Festuco-Brometea, FPe: Festuco-Puccinellietea, FPi: Festuco-Puccinellietalia, Fvg: Festucetea vaginatae, Fvl: Festucetalia valesiacae, GA: Galio-Alliarion, MoA: Molinio-Arrhenatherea, NC: Nardo-Callunetea, OCn: Orno-Cotinion, PP: Pulsatillo-Pinetea, Prs: Prunion spinosae, Pru: Prunetalia, Pte: Phragmitetea, QF: Querco-Fagea, Qia: Quercetalia pubescentis-petraeae, Qpp: Quercetea pubescentis-petraeae, Qrp: Quercetea robori-petraeae, S: sum (in total), Sal: Salicion albae, Sea: Secalietea, SFe: Seslerio-Festucion pallentis, Spu: Salicetea purpureae, TAc: Tilio-Acerion, US: 
The differential species of the Poo nemoralis-Tilietum cordatae are definitely acidophilous plants in the herb layer, such as Vaccinium myrtillus, Deschampsiaflexuosa, Teucrium scorodonia. These species are absent from the

Table 5

Lichen, moss and hepatic species combination of northern and southern type of the Roso pendulinae-Tilietum cordatae

\begin{tabular}{|c|c|}
\hline Northern type (serial number: 2394) & Southern type (serial number: 2369 ) \\
\hline \multicolumn{2}{|c|}{ Lichens (35 species) } \\
\hline $\begin{array}{l}\text { On basalt: } \\
\text { Diploschistes scruposus (Schreber) Norman } \\
\text { Trapelia coarctata (Sm.) Choisy }\end{array}$ & $\begin{array}{l}\text { On basalt: } \\
\text { Acarospora fuscata (Nyl.) Th. Fr. } \\
\text { Diploschistes scruposus (Schreber) Norman } \\
\text { Hypocenomyce scalaris (Ach. ex Lilj.) Choisy } \\
\text { Lasallia pustulata (L.) Mérat } \\
\text { Lecanora polytropa (Ehrh. ex Hoffm.) } \\
\text { Rabenh. } \\
\text { Lecanora rupicola (L.) Zahlbr. } \\
\text { Lecidea fuscoatra (L.) Ach. } \\
\text { Lepraria caesioalba (B. de Lesd.) Laundon } \\
\text { Leproloma membranaceum (Dickson) Vainio } \\
\text { Melanelia disjuncta (Erichsen) Essl. } \\
\text { Melanelia sorediata (Ach.) Goward et Ahti } \\
\text { Neofuscelia pulla (Ach.) Essl. } \\
\text { Peltigera praetextata (Flörke ex Sommerf.) } \\
\text { Zopf } \\
\text { Porpidia glaucophaea (Körber) Hertel et } \\
\text { Knoph } \\
\text { Rhizocarpon geographicum (L.) DC. } \\
\text { Scoliciosporum umbrinum (Ach.) Arnold } \\
\text { Xanthoparmelia conspersa (Ehrh. ex Ach.) } \\
\text { Hale } \\
\text { Xanthoparmelia somloensis (Gyelnik) Hale }\end{array}$ \\
\hline $\begin{array}{l}\text { On soil: } \\
\text { Cladonia coccifera (L.) Willd. } \\
\text { Cladonia fimbriata (L.) Fr. } \\
\text { Cladonia pyxidata (L.) Hoffm. } \\
\text { Cladonia rangiferina (L.) Weber ex Wigg. } \\
\text { Cladonia squamosa (Scop.) Hoffm. }\end{array}$ & $\begin{array}{l}\text { On soil: } \\
\text { Cladonia cenotea (Ach.) Schaerer } \\
\text { Cladonia macilenta Hoffm. } \\
\text { Cladonia rangiferina (L.) Weber ex Wigg. } \\
\text { Cladonia squamosa (Scop.) Hoffm. }\end{array}$ \\
\hline $\begin{array}{l}\text { On trunk (bark): } \\
\text { Cladonia coniocraea auct. } \\
\text { Lecanora conizaeoides Nyl. ex Crombie } \\
\text { Lecidella elaeochroma (Ach.) Choisy } \\
\text { Lepraria incana (L.) Ach. } \\
\text { Melanelia glabratula (Lamy) Essl. } \\
\text { Parmelina tiliacea (Hoffm.) Hale } \\
\text { Phlyctis argena (Sprengel) Flotow }\end{array}$ & $\begin{array}{l}\text { On trunk: } \\
\text { Hypocenomyce scalaris (Ach. ex Lilj.) Choisy } \\
\text { Micarea denigrata (Fr.) Hedl. } \\
\text { Parmeliopsis ambigua (Wulfen) Nyl. }\end{array}$ \\
\hline
\end{tabular}




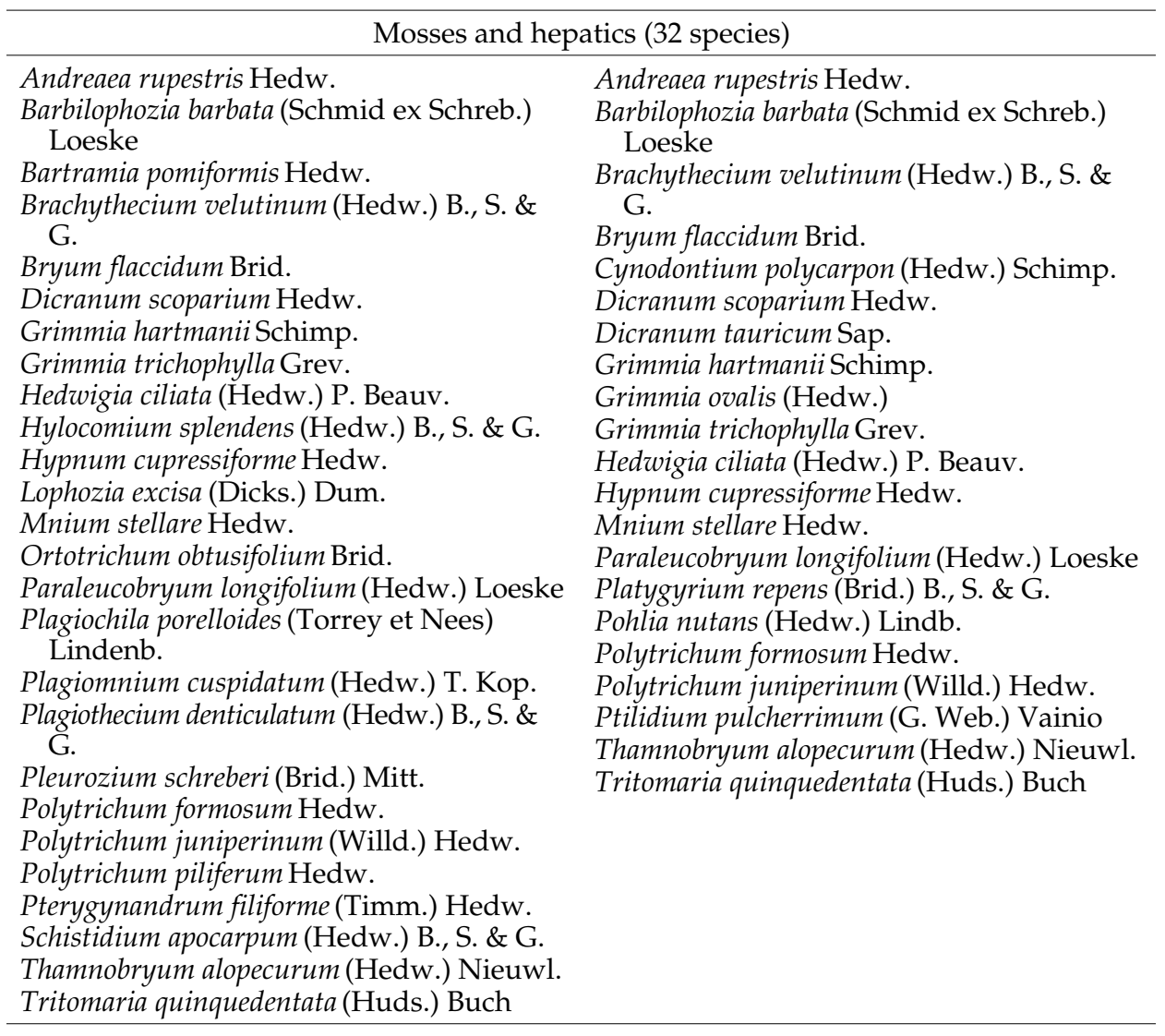

Nógrád-Gömör area or the occurrence of these acidophilous elements has not been proved.

The negative differential species of the Roso pendulinae-Tilietum cordatae against the Poo nemoralis-Tilietum cordatae in the canopy is the Fraxinus excelsior (Bartsch and Bartsch 1940), which occurs at several localities but is always absent on the thick periglacial block fields of the Cerová Vrchovina. The ash occurs only on thinner scree slopes, where the canopy is better developed and more closed. In these habitats the species combination of the shrub, herb and moss layers recall rather the flora of the submontane beech woods. The conifers are absent in the canopy because of certain vegetation historical causes (Zólyomi 1952). The positive differential species of the Roso pendulinae-Tilietum cordatae are the continental elements of the oak woods and rocky grasslands, which are widely distributed in the Matricum (e.g. Spiraea media, Cotoneaster matrensis, Rosa spinosissima, Euonymus verru- 
cosus in the shrub layer), and the elements of the montane-alpine region, which occur in the Alps and the Carpathians, such as Rosa pendulina (Meusel and Jäger 1992). Asplenium septentrionale, Sedum maximum, Solanum dulcamara and Andreaea rupestris are characteristic in the herb and moss layers, but they are absent in the Poo nemoralis-Tilietum cordatae (Firbas and Sigmond 1928).

The characteristic species combination of the Roso pendulinae-Tilietum cordatae ass. nova (Table 4, relevés 1-15; nomenclatural type: Table 4, relevé 1 ) is the following: Tilia cordata in the canopy, Sorbus aucuparia, Betula pendula, Populus tremula occurring both in the canopy and shrub layer, Rosa pendulina, Sambucus racemosa, Rubus idaeus, Spiraea media in the shrub layer, Asplenium septentrionale, Dryopteris dilatata, Solanum dulcamara, Luzula luzuloides, Festuca altissima in the herb layer, Andreaea rupestris, Dicranum tauricum, Tritomaria quinquedentata and Cladonia rangiferina in the moss layer. Other characteristic species are Tilia platyphyllos in the canopy, Euonymus verrucosus, Lonicera xylosteum in the shrub layer, Dryopteris filix-mas, Polypodium vulgare, Poa nemoralis in the herb layer, Barbilophozia barbata, Dicranum scoparium, Paraleucobryum longifolium, Polytrichum spp., Hedwigia ciliata and Diploschistes scruposus in the moss layer. There are two subtypes of this community, one in southern (e.g. serial number 2394) and one in northern exposition (e.g. serial number 2369) (Table 4). In these cases the presence/absence of the Tilia species and the moss layer show the greatest ecological difference (Table 5).

There are stands with the same species combination in the Matra Mts (Kékestető-Saskő) and according to the relevés of Simon (1977) in the Zemplén Mts. The characteristic and differential species are present in the Börzsöny Mts too, but they do not coexist within one association (Nagy J. ex verb.).

\section{Raunkiaer's life form categories and flora elements}

According to Raunkiaer's life form categories there are characteristic differences between the Roso pendulinae-Tilietum cordatae (Cerová Vrchovina) and the Mercuriali-Tilietum (Keszthely Mts, Bakony Mts and Mátra Mts). The mega-meso-, the micro- and the nanophanerophyton species $(\mathrm{MM}, \mathrm{M}, \mathrm{N})$ are represented with high frequency in the block forest, while geophytes play a restricted role (Fig. 2). The presence of the latter on scree slopes gradually decreases from the Transdanubian Mts to the Northern Hungarian Mts. The thicker debris cover, made up of larger blocks, the re- 


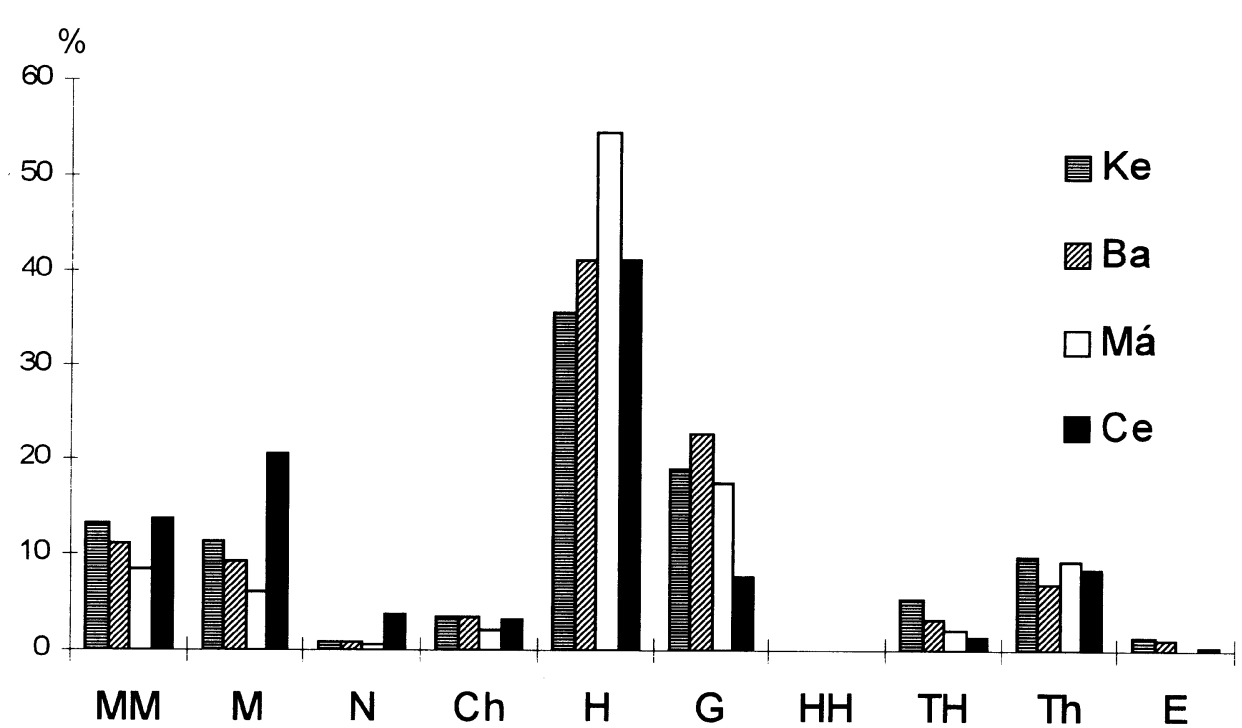

Fig. 2. Raunkiaer's life form categories. Ke: Keszthely Mts. Ba: Bakony Mts. Má: Mátra Mts. Ce: Cerová Vrchovina

stricted soil development and the increasing climatic extremities are considered to be in the background of this observation. In these areas the January mean temperature drops below -3 to $-4^{\circ} \mathrm{C}$ and the frost is not unusual in spring. Under these circumstances the best growing period for the early vernal geophytes is postponed to the time when the trees come into leaf, but at that time the light and soil conditions are already unfavourable for these species (Kevey 1978). This phenomenon may be amplified by the fact that the locations of the frost traps coincide with those where the thin soil accumulates. The snow may remain among the blocks till May. Only the chasmophytes, lichens, mosses, ferns and some frost-tolerant tree and shrub species can stand these conditions.

There are some characteristic differences between the block forest and the lime-dominated scree forest (Mercuriali-Tilietum) considering geo elements and distribution types. The circumpolar, boreal and subalpine species show their maximum in the Roso pendulinae-Tilietum cordatae. These plants are the "gap" species of the montane beech woods. Furthermore, European and submediterranean elements play a decreasing role in this community (Fig. 3). 


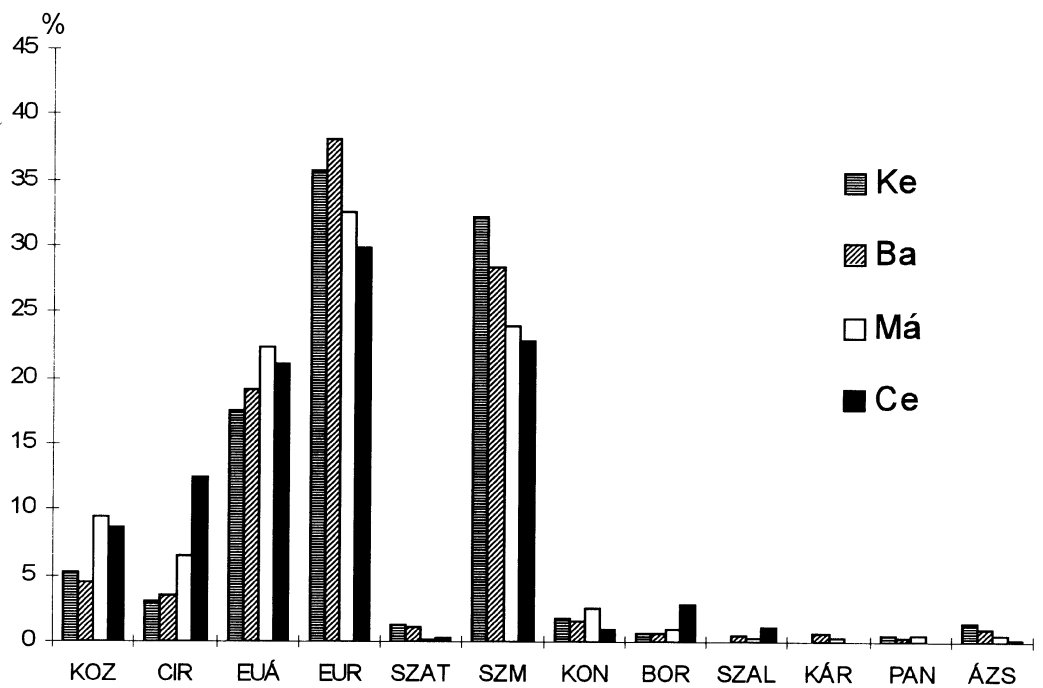

Fig. 3. Geo elements and distribution types. KOZ: cosmopolitan, CIR: circumpolar, EUÁ: Eurasian, EUR: European, SZAT: subatlantic, SZM: submediterranean, KON: continental, BOR: boreal, SZAL: subalpine, KÁR: Carpathian endemic, PAN: Pannonian endemic, ÁZS:

Asian, Ke: Keszthely Mts, Ba: Bakony Mts, Má: Mátra Mts, Ce: Cerová Vrchovina

\section{Relative ecological indicator values}

Again Mercuriali-Tilietum relevés of three Hungarian mountains (Keszthely Mts, Bakony Mts, Mátra Mts) and the block forest relevés of the Cerová Vrchovina were examined using the relative ecological indicator

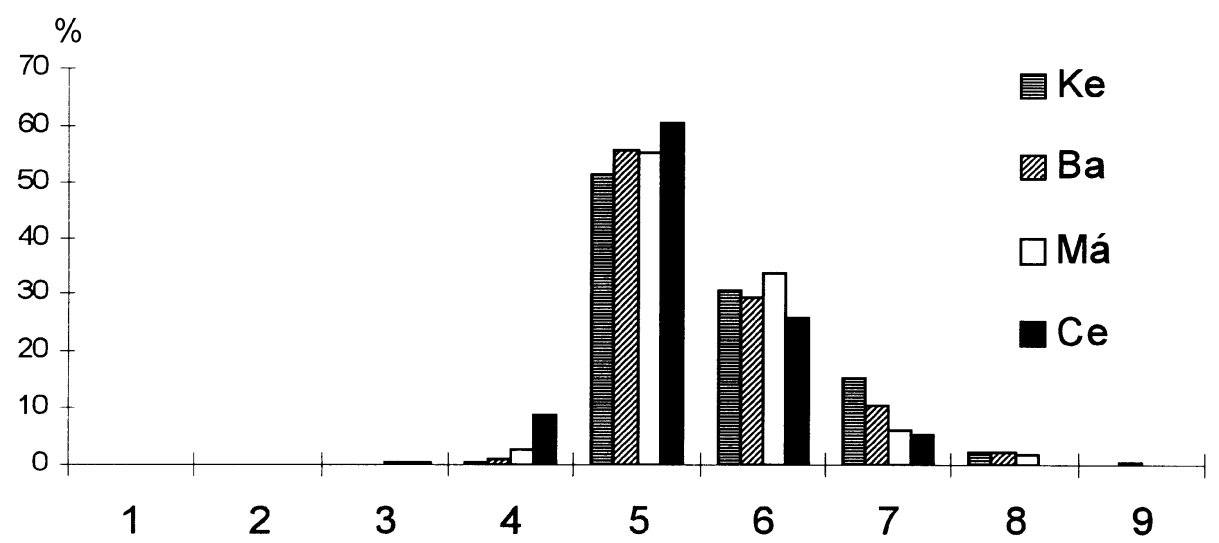

Fig. 4. Relative heat indicator (TB) spectrum. Ke: Keszthely Mts, Ba: Bakony Mts, Má: Mátra Mts, Ce: Cerová Vrchovina 


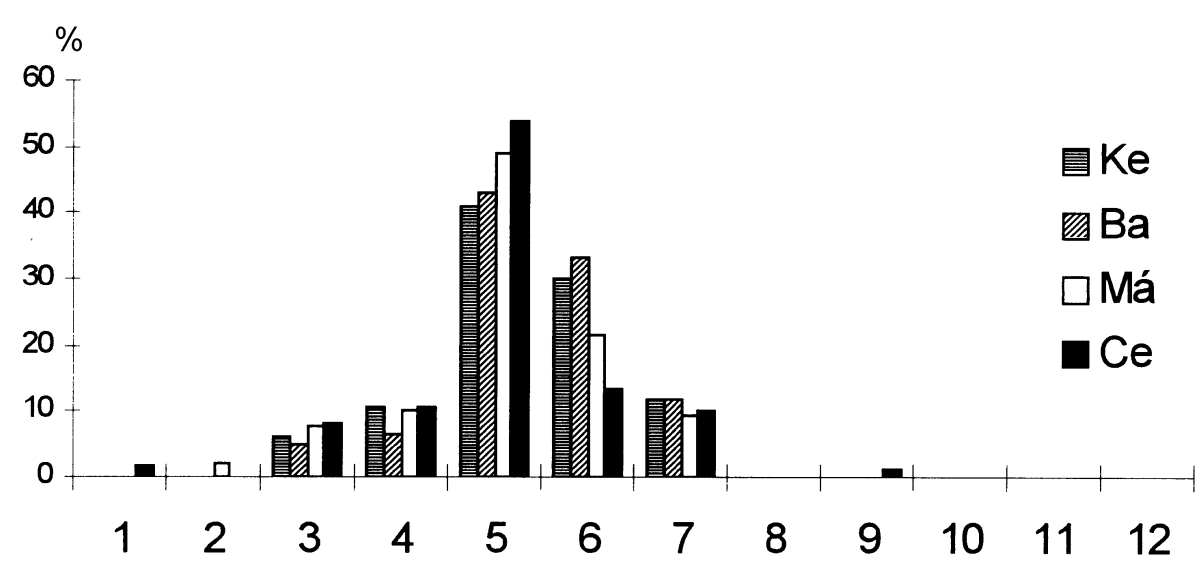

Fig. 5. Relative soil moisture indicator (WB) spectrum. Ke: Keszthely Mts, Ba: Bakony Mts, Má: Mátra Mts, Ce: Cerová Vrchovina

values of Borhidi (1993). Based on the relative temperature figures (TB) it can be stated that in the case of block forest (Fig. 4) the average values shifted towards the cooler, higher zones, which refers to the presence of relict species from the subboreal period.

According to the soil moisture or water table values (WB), a minor microclimatic bipolarity can be seen due to the heterogeneous surface of the block fields (blocks and gaps), so both xerophilous elements and plants of wet habitats are present, while they are absent in lime scree forests (Fig. 5).

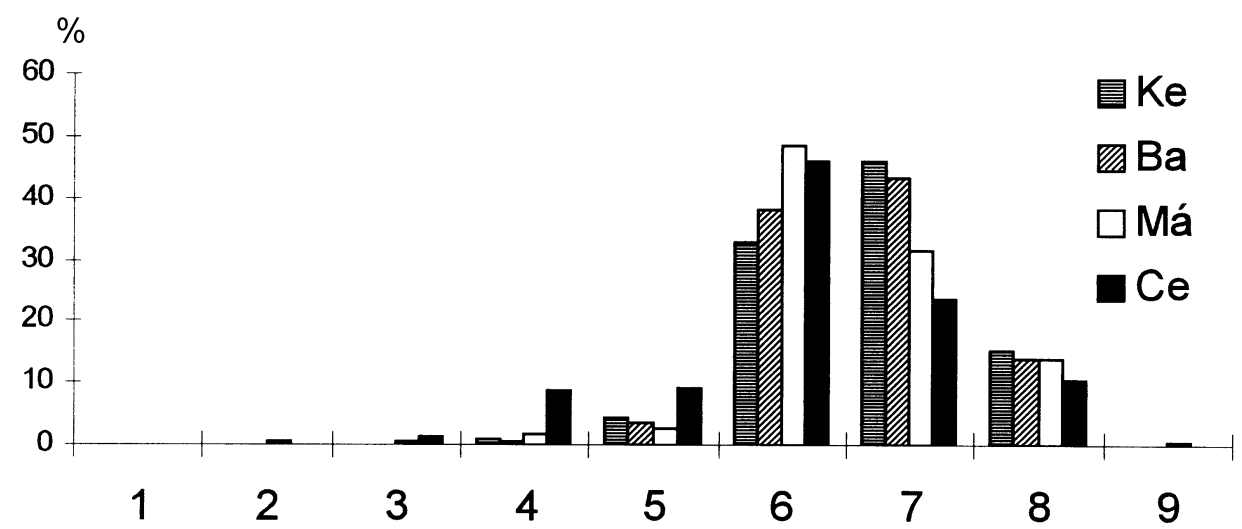

Fig. 6. Relative soil reaction indicator (RB) spectrum. Ke: Keszthely Mts, Ba: Bakony Mts, Má: Mátra Mts, Ce: Cerová Vrchovina 
Comparing the two mentioned associations, the acidic soil reaction (RB) categories appear more frequently in the block forest (Fig. 6). It is the result of the siliceous substrate (basalt) and the slow soil development.

There are no significant differences between the Mercuriali-Tilietum and the Roso pendulinae-Tilietum cordatae considering the ammonia and nitrate supply of the habitats (NB), except that the spectrum is wider in the block forest and the extreme categories show the highest values (Fig. 7). It may be the consequence of the heterogeneous surface and the open canopy.

In the case of the relative light intensity during the summer (LB), the ratio of light and halflight plants is higher in the block forest than in the Mercuriali-Tilietum, which can be explained by the open canopy of the former community (Fig. 8).

\section{Cluster analysis}

For this analysis 74 relevés from six mountains in the Carpathian Basin (Keszthely Mts, Bakony Mts, Gerecse Mts, Mátra Mts, Zemplén Mts and Cerová Vrchovina) were included. Distances were calculated using binary data, because the presence/absence of the species is thought to be a more distinctive character between Mercuriali-Tilietum and the Roso pendulinae-Tilietum cordatae associations than their coverage values. Since there

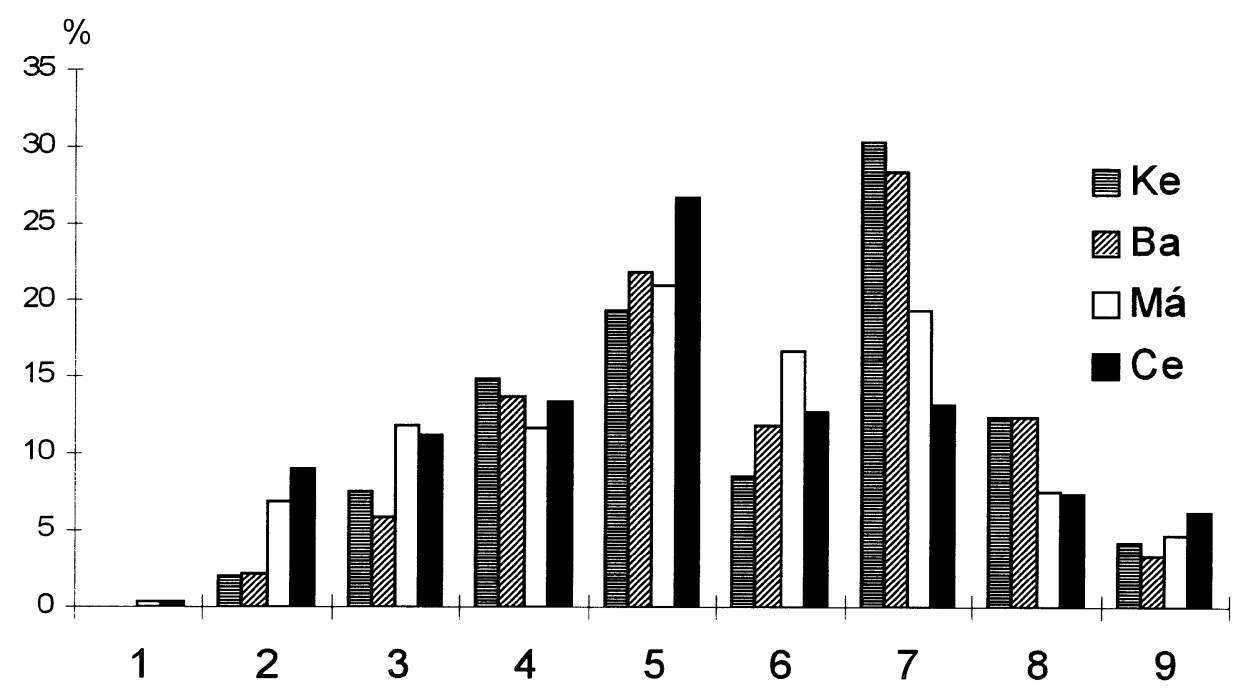

Fig. 7. Relative nutrient supply (NB) spectrum. Ke: Keszthely Mts, Ba: Bakony Mts, Má: Mátra Mts, Ce: Cerová Vrchovina 


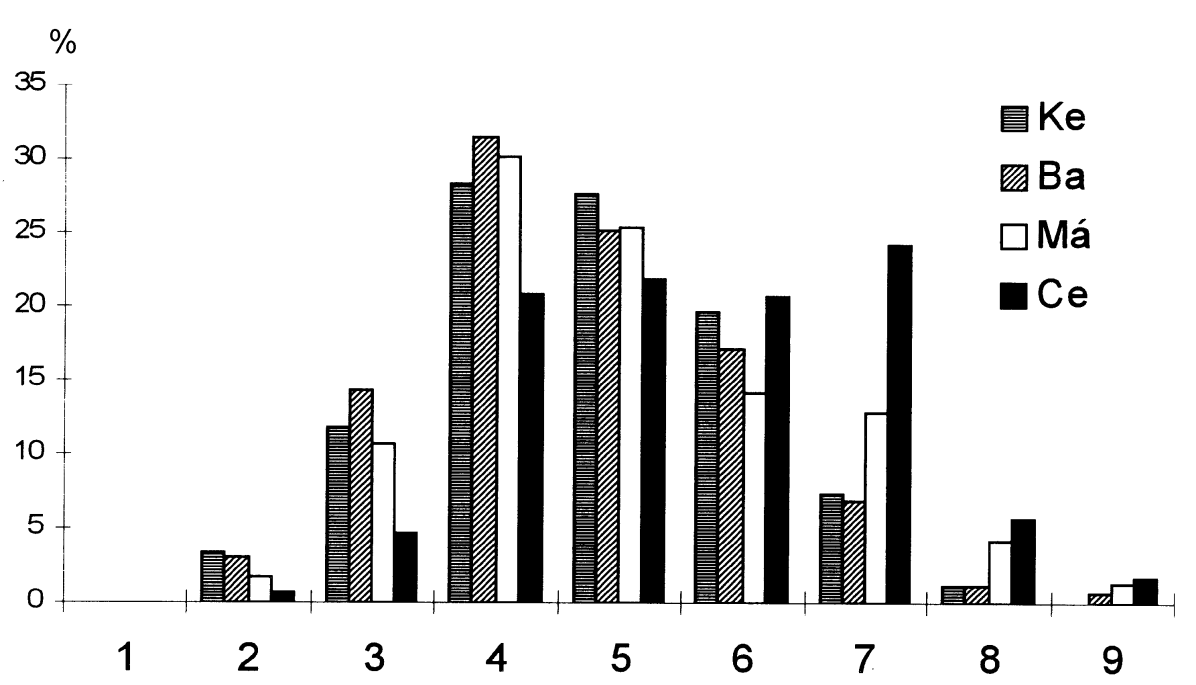

Fig. 8. Relative light intensity (LB) spectrum. Ke: Keszthely Mts, Ba: Bakony Mts, Má: Mátra Mts, Ce: Cerová Vrchovina

were significant differences between the examined forest communities in species combination of the canopy, the shrub and herb layer, distance calculations for all layers were also carried out. Only one dendrogram is shown here (Fig. 9), since all applicable algorithms gave quite similar results.

The relevés of the Northern Hungarian Mts (Mátra Mts, Zemplén Mts and Cerová Vrchovina) gathered in one group and were isolated from the relevés of Transdanubian Mts (Keszthely Mts, Bakony Mts, Gerecse Mts). It may be the consequence of the abiotic background pattern, which is different on the two sides of the so-called "Central Danubian flora boundary", described by Zólyomi (1942) which divides the Pannonicum province into two sectors: one with milder annual temperature and precipitation (Transdanubian region) and one with more extreme climate (northeast from the river Danube). This division is amplified by the geological heterogeny, since mostly sedimental (limestone, dolomite) rocks build up the Transdanubian Mts, while there is mostly volcanic (andesite, rhyolite) substrate in the Northern Hungarian Mts.

The Roso pendulinae-Tilietum cordatae stands separate from the Mercuriali-Tilietum relevés of the Mátra and Zemplén Mts on the left side of the dendrogram. Only one stand from the Zemplén Mts is similar to those of the block forests from the Cerová Vrchovina, which may show the neigh- 


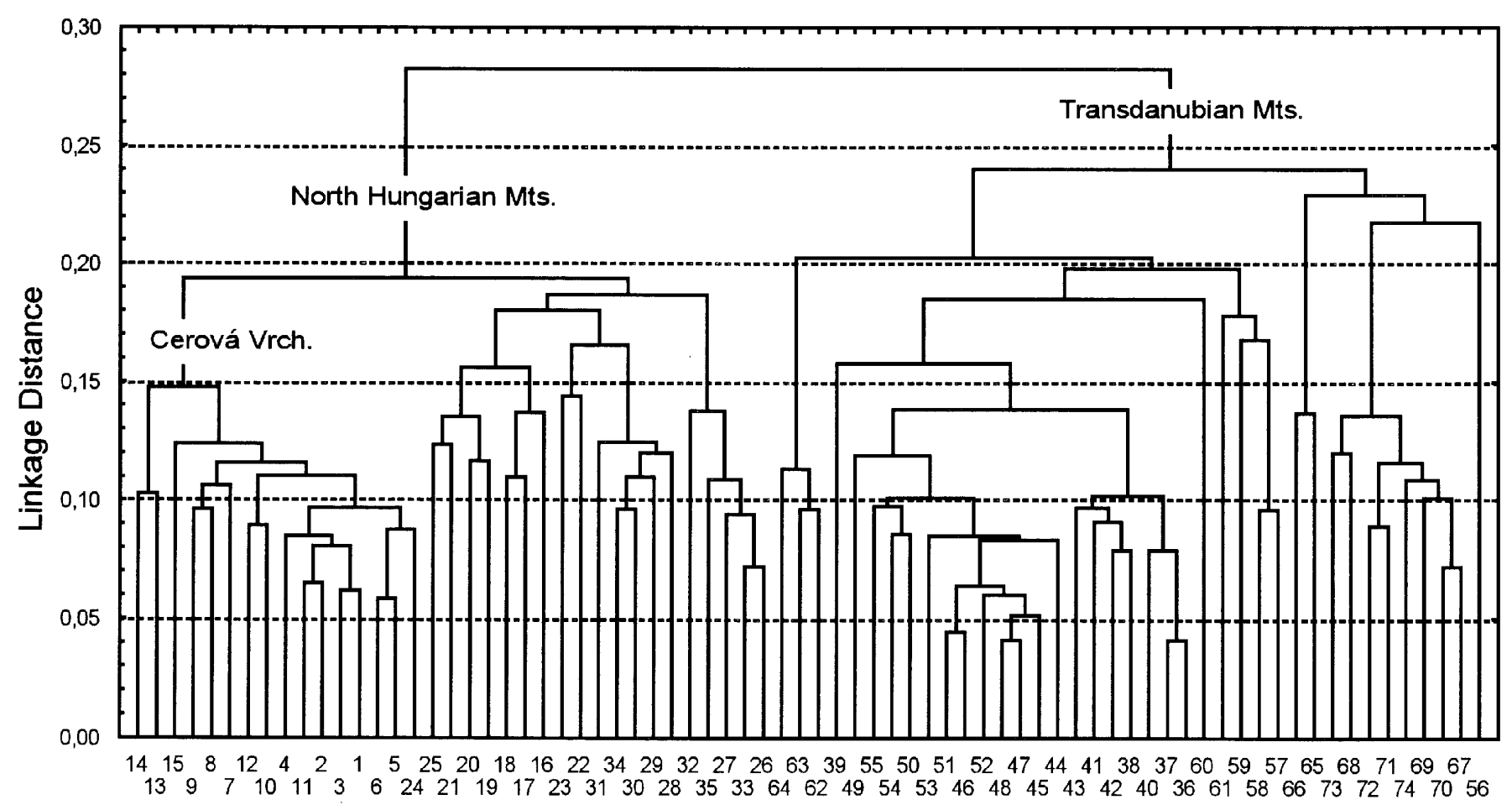

Fig. 9. Dendrogram for the Mercuriali-Tilietum and Roso pendulinae-Tilietum cordatae relevés on periglacial stony slopes in the Matricum (weighted pair-group average, percent disagreement). 1-15: Cerová Vrchovina, 16-25: Zemplén Mts, 26-35: Mátra Mts 36-55: Gerecse Mts, 56-64: Bakony Mts, 65-74: Keszthely Mts 
bourhood of the Carpathians and the common historical processes of the vegetation.

This separation seems to prove the results of the previous statistical analyses (phytotaxonomic characterization, Raunkiaer's life form categories, flora elements and relative ecological indicator values).

Acknowledgements - This research was supported by the PhD program of the Botanical Department of the University of Pécs and the Research Group for Biological Adaptation of the Hungarian Academy of Sciences (University of Pécs). The authors thank László Lókös and Péter Ódor, who collected and determined the lichens and moss species of the Cerová Vrchovina and József Nagy for his unpublished Mercuriali-Tilietum relevés from the Börzsöny Mts.

\section{REFERENCES}

Bartha, D., Kevey, B., Morschhauser, T. and Pócs, T. (1995): Hazai erdőtársulásaink. - Tilia 1: $8-85$.

Bartsch, J. and Bartsch, M. (1940): Vegetationskunde des Schwarzwaldes. - Gustav Fischer Verlag, Jena.

Borhidi, A. (1993): A magyar flóra szociális magatartás típusai, természetességi és relatív ökológiai értékszámai. - A Környezetvédelmi és Területfejlesztési Minisztérium Természetvédelmi Hivatala és a Janus Pannonius Tudományegyetem kiadványa, Pécs.

Borhidi, A. (1995): Social behavior types, the naturalness and relative ecological indicator values of the higher plants in the Hungarian flora. - Acta Bot. Hung. 39: 97-181.

Borhidi, A. (ed.) (1996): Critical revision of the Hungarian plant communities. - Janus Pannonius University, Pécs.

Braun-Blanquet, J. (1928): Pflanzensoziologie. - Julius Springer Verlag, Berlin, 330 pp.

Corley, M. F. V. and Crundwell, A. C. (1991): Additions and amendments to the mosses of Europe and the Azores. - J. Bryol. 16: 337-356.

Corley, M. F. V., Crundwell, A. C., Düll, R., Hill, M. O. and Smith, A. J. E. (1981): Mosses of Europe and the Azores, an annotated list of species, with synonyms from the recent literature. - J. Bryol. 11: 609-689.

Debreczy, Zs. (1981): Növényvilág a Balaton körüll. - In: Illés, I. (ed.): Tavunk, a Balaton. Natura, Budapest, pp. 75-120.

Dostál, J. and Cervenka, M. (1991): Velky Kluc na urcovanie vyssich rastlin I. - Slovenské Pedagogické Nakladatelstvo, Bratislava, 775 pp.

Fekete, G. (1964): A Bakony növénytakarója. (A Bakony cönológiai-növényföldrajzi képe). In: Papp, J. (ed.): A Bakony természettudományi kutatásának eredményei I. A Veszprém Megyei Múzeumok Igazgatósága, Veszprém, pp. 7-40.

Fekete, G. and Járai-Komlódi, M. (1962): Die Schuttabhangwälder der Gerecse- und BakonyGebirge. - Ann. Univ. Sci. Budapest. Rolando Eötvös nom., Sect. Biol.5: 115-129. 
Fekete, G., Molnár, Zs. and Horváth F. (eds) (1997): Nemzeti Biodiverzitás-monitorozó Rendszer II. A magyarországi élőhelyek leírása, határozója és a Nemzeti Élóhely-osztályozási Rendszer. - Magyar Természettudományi Múzeum, Budapest.

Firbas, F. and Sigmond, H. (1928): Vegetationsstudien auf dem Donnersberge im Böhmischen Mittelgebirge. - Lotos 76: 113-176.

Gábris, Gy. (1995): Éghajlati felszinalaktan I. (Periglaciális geomorfológia). - Nemzeti Tankönyvkiadó, Budapest.

Grolle, R. (1983): Hepatics of Europe including the Azores: an annotated list of species, with synonyms from the recent literature. - J. Bryol. 12: 403-459.

Horánszky, A. (1964): Die Wälder des Szentendre-Visegrader Gebirges. - Akadémiai Kiadó, Budapest, $288 \mathrm{pp}$.

Horváth, F., Dobolyi, Z. K., Morschhauser, T., Lőkös, L., Karas, L. and Szerdahelyi, T. (1995): Flóra adatbázis 1.2 Taxonlista és attribútum-állomány.- Vácrátót, 267 pp.

Horváth, G., Munkácsy, B., Pintér, Z., Csiky, J., Karancsi, Z. and Prakfalvi, P. (1997): A Medves. - Földr. Ért. 46(3-4): 217-248.

Isépy, I. (1968): Szurdokerdők és törmeléklejtő-erdők a Vértes-hegységben. - Bot. Közlem. 55: 199-204.

Jakucs, P. (1961): Az Északi-középhegység keleti felének növényzete. - Földr. Ért. 10(4): 357-377.

Jugovics, L. (1971): Észak-magyarországi - Salgótarján-környéki - bazaltterületek. - Földt. Int. Évi jel. 1968-ról, pp. 145-165.

Kevey, B. (1978): Az Allium ursinum L. magyarországi elterjedése. - Bot. Közlem. 65(3): $165-175$.

Kevey, B. (1993): A Szigetköz ligeterdeinek összehasonlító-cönológiai vizsgálata. - Kandidátusi értekezés, MTA, Budapest, (kézirat).

Kevey, B. (1997): A Nyugati-Mecsek szurdokerdei (Scutellario altissimae-Aceretum (Horvát A. O. 1958) Soó et Borhidi in Soó 1962). - Studia Phytologica Jubilaria, JPTE Növénytani Tanszék, Pécs, pp. 75-99.

Kevey, B. and Borhidi, A. (1998): Top-forest (Aconito anthorae-Fraxinetum orni) a special ecotonal case in the phytosociological system (Mecsek Mts, South Hungary). - Acta Bot. Hung. 41(1-4): 27-121.

Kovács, J. A. and Takács, B. (1995): A Balatonvidék bazaltvulkáni növényzetének sajátosságairól. - Kanitzia 3: 51-97.

Kovács, M. (1968): Die Acerion pseudoplatani-Wälder (Mercuriali-Tilietum und PhyllitidiAceretum) des Mátra-Gebirges. - Acta Bot. Acad. Sci. Hung. 14(3-4): 331-350.

Kun, A. (1998): Gondolatok a reliktum kérdésről. (Kontinentális reliktumjellegü vegetációmozaikok a Magyar Középhegységben). - In: Csontos, P. (ed.): Sziklagyepek szünbotanikai kutatása. Scientia Kiadó, Budapest, pp. 197-212.

Mayer, H. (1984): Wälder Europas. - Gustav Fischer Verlag, Stuttgart, New York.

Meusel, H. and Jäger, E. J. (eds) (1992): Vergleichende Chorologie der Zentraleuropäischen Flora I. - Gustav Fischer Verlag, Jena, Stuttgart, New York.

Michalko, J. (ed.) (1987): Geobotanical map of CSSR. Slovak Socialist Republik. - VEDA Publishing House of the Slovak Academy of Sciences, Bratislava.

Mucina, L., Grabherr, G. and Wallnöfer, L. (1993): Die Pflanzengesellschaften Österreichs III. Wälder und Gebüsche. - Gustav Fischer Verlag, Jena, Stuttgart, New York.

Nagy, J. (1999): A Börzsöny-hegység szurdokerdei. - Kitaibelia 4(2): 301-310.

Oberdorfer, E. (1957): Süddeutsche Pflanzengesellschaften.- Gustav Fischer Verlag, Jena.

Pott, R. (1995): Die Pflanzengesellschaften Deutschlands.- Verlag Eugen Ulmer, Stuttgart. 
Sen, D. N. (1961): Self-saprophytism in roots of Tilia cordata L. - Preslia 33: 36-40.

Simon, T. (1977): Vegetationsuntersuchungen im Zempléner Gebirge. - Akadémiai Kiadó, Budapest.

Simon, T. (1992): A magyarországi edényes flóra határozója. (Field guide to the Hungarian vascular flora). - Tankönyvkiadó, Budapest, 892 pp.

Soó, R. (1960): Magyarország új florisztikai-növényföldrajzi felosztása. - MTA Biol. Csop. Közlem. 4: 43-70.

Szmorad, F. (1994): A Kőszegi-hegység erdőtársulásai. - In: Bartha, D. (ed.): A Kőszegi-hegység vegetációja. Kőszeg-Sopron, pp. 106-132.

Szujkó-Lacza, J. (1967): Beiträge zur Kenntnis der Pflanzengesellschaften des BörzsönyGebirge I. - Annls hist.-nat. Mus. natn. Hung. 59: 147-161.

Verseghy, K. (1994): Magyarország zuzmóflórájának kézikönyve. - MTM, Budapest, 415 pp.

Vojtkó, A. (1990): A Központi-Bükk déli előterének vegetációja. - Fol. Hist.-nat. Mus. Matr. 15: 27-36.

Vojtkó, A. (1995): A Naszály hegy növénytársulásai. - Acta Acad. Agr. Nova Ser. 21(1): 355-361.

Vojtkó, A. (1996): Szarvaskő vegetációja (Bükk hegység) és sziklagyepjeinek fitocönológiája. Bot. Közlem. 83(1-2): 7-24.

Zólyomi, B. (1942): A középdunai flóraválasztó és a dolomitjelenség. (Die MitteldonauFlorenscheide und das Dolomitphänomen). - Bot. Közlem. 39(5): 209-234.

Zólyomi, B. (1952): Magyarország növénytakarójának fejlődéstörténete az utolsó jégkorszaktól. - MTA Biol. Oszt. Közlem. 1: 491-530

Zólyomi, B. (1958): Budapest és környékének természetes növénytakarója. (The natural vegetation of Budapest and its environs). - In: Pécsi, M. (ed.): Budapest természeti képe. Akadémiai Kiadó, Budapest, pp. 509-642.

Zólyomi, B. (ed.) (1967): Guide der Excursionen des Internationalen Geobotanischen Symposiums, Ungarn. - Eger-Vácrátót. 